\title{
A Review on the Behavioral Tests for Learning and Memory Assessments in Rat
}

\section{Fahimeh Zavvari $^{1,2}$, Fariba Karimzadeh ${ }^{3 *}$}

${ }^{1}$ Shefa Neuroscience Research Center, Khatam Alanbia Hospital, Tehran, Iran

${ }^{2}$ Department of Physiology, School of Medicine, Iran University of Medical Sciences, Tehran, Iran

${ }^{3}$ Cellular and Molecular Research Center, School of Medicine, Iran University of Medical Sciences, Tehran, Iran

\section{Article Info:}

\section{ABSTRACT}

Introduction: Memory is a fundamental mental process and without memory we are capable of nothing, except simple reflexes and stereotype behaviors. The memory deficits followed by head injury, stroke, psychiatric and neurodegenerative diseases are common. Learning and memory is one of the main subjects that have been studied in the field of neuroscience. Considering ethical issues, humans can rarely be used as experimental model in medical researches. Thus, different kind of experimental tests have been designed that assess learning and memory deficits in animals in order to find effective treatment strategies. The purpose of this review was to evaluate the most commonly used tests for memory and learning in rats, including open field, habituation, Y-maze, step through passive avoidance test, step-down inhibitory avoidance task, the Morris water maze, active avoidance test, 8-arm radial maze, and novel object recognition test. Conclusion: Different tests have been designed to evaluate various kinds of memory (depends on function and duration). Behavioral tests in the field of learning and memory have been beneficial to improve our understanding of information processing in healthy and damaged brain as well as the physiological and psychological aspects of memory disorders. This may be helpful to design new therapies for learning and memory deficits in patients with neurological disorders.

Key words:

1. Learning

2. Memory

3. Rats

*Corresponding Author: Fariba Karimzadeh

E-mail: Fariba_karimzade@yahoo.com 


\title{
مرورى بر آزمونهاى رفتارى ارزيابى حافظه و يادگيرى در موش صحرايى
}

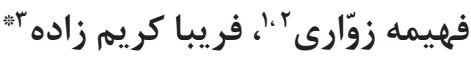 \\ 'مركز تحقيقات علوم اعصاب شفا، بيمارستان خاتمالانبياء، تهران، ايران

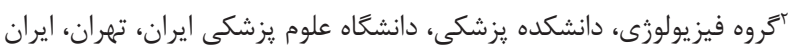

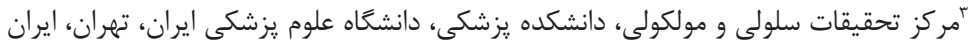

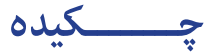

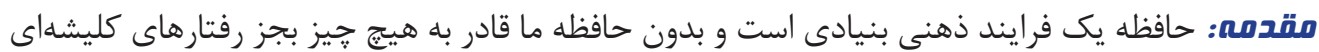

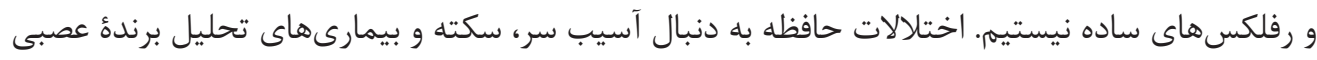

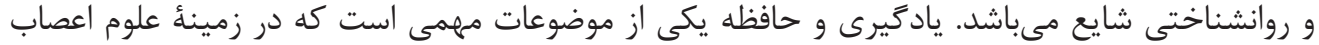

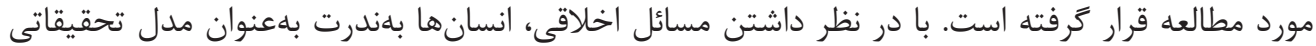

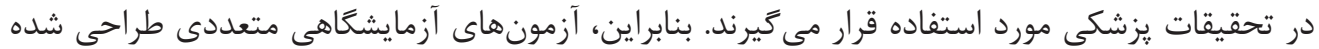

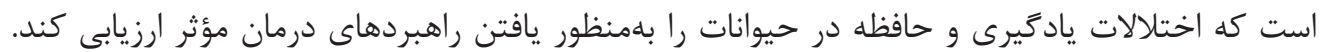

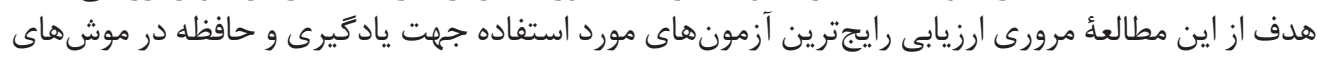

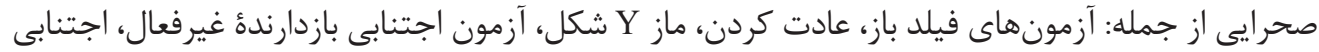

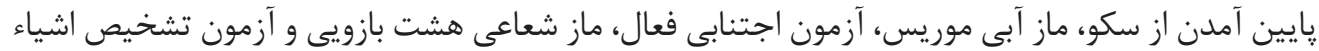

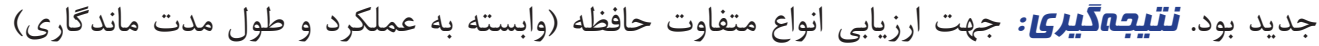

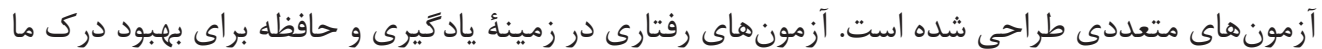

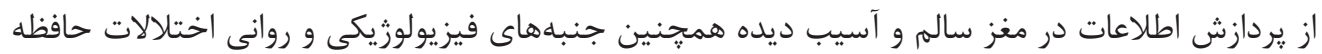

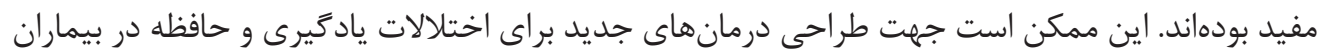

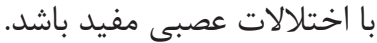




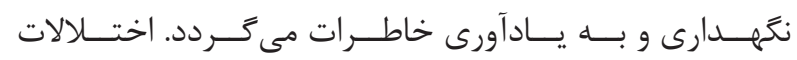

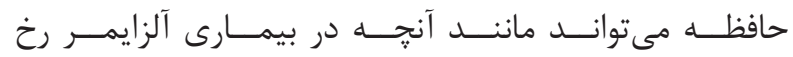

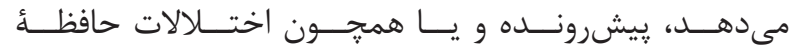

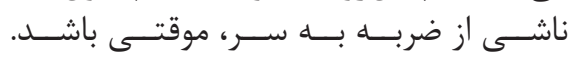

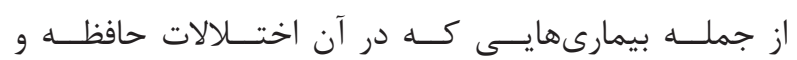

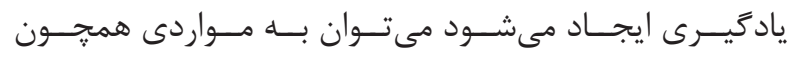

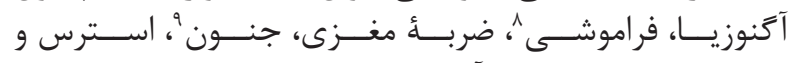

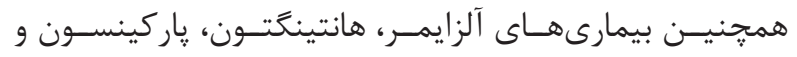

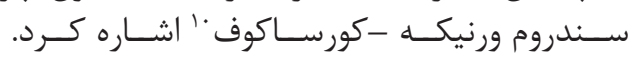

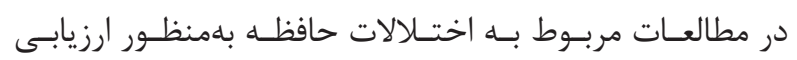

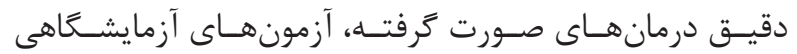

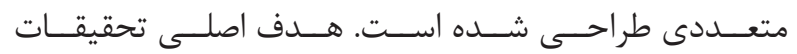

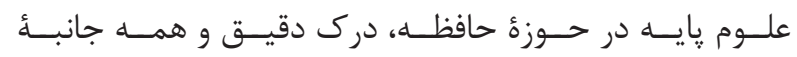

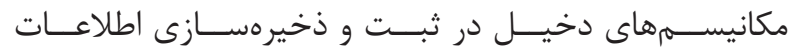

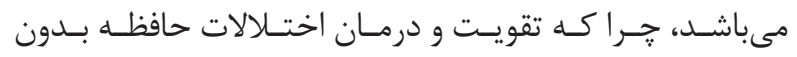

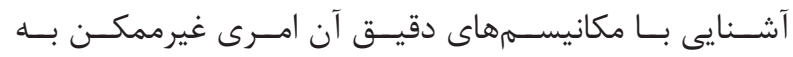

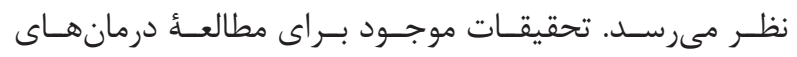

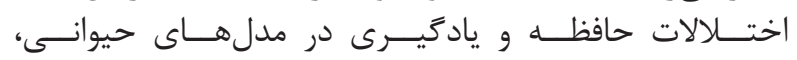

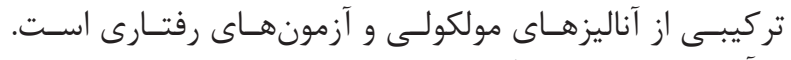

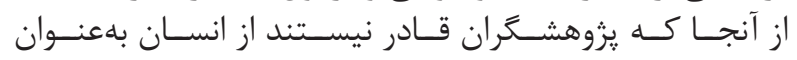

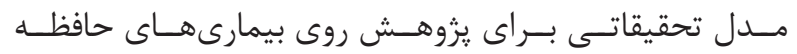

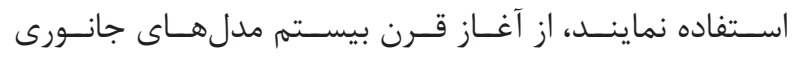

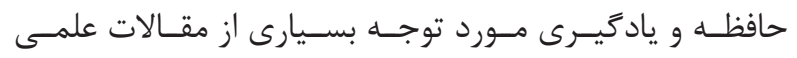

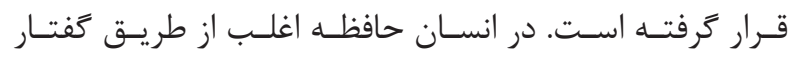

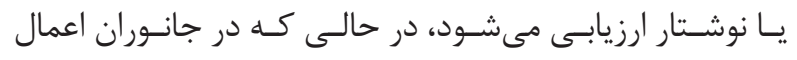

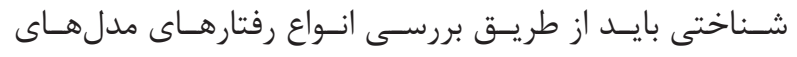

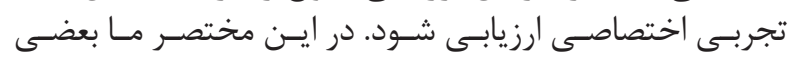

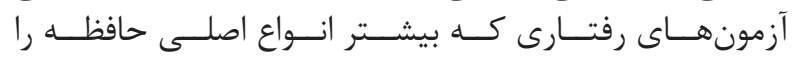

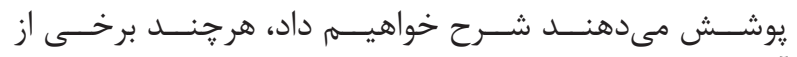

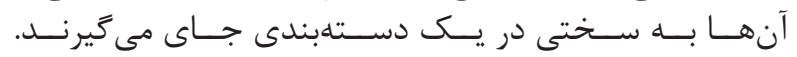

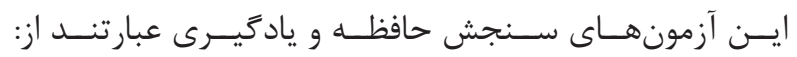

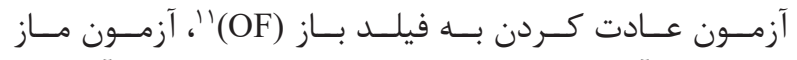

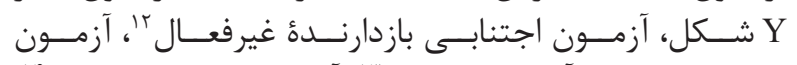

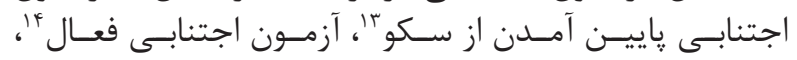

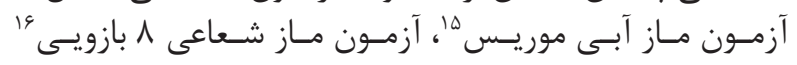

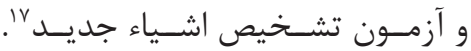

$$
\begin{aligned}
& \text { آزمون عادت كردن به زمينه باز }
\end{aligned}
$$

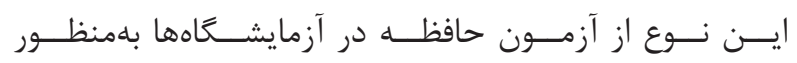

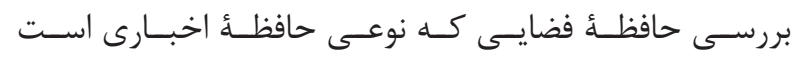

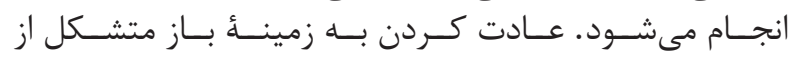

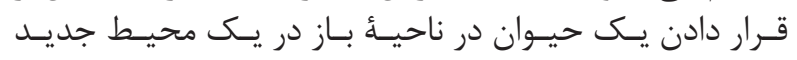

${ }^{1}$ Encoding

${ }^{2}$ Storage

${ }^{3}$ Retrieval/recall

${ }^{4}$ Explicit memory

${ }^{5}$ Declarative

${ }^{6}$ Implicit memory

${ }^{7}$ Non declarative

${ }^{8}$ Amnesia

${ }^{9}$ Dementia

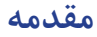

بــه قابليـت مغــز در ذخيــره، نخــهدارى و يـادآورى اطلاعـات

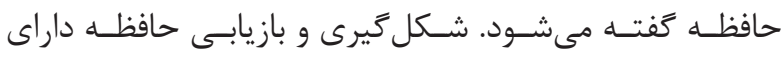

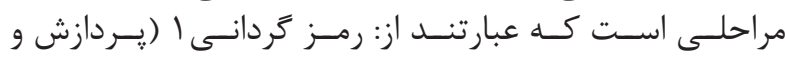

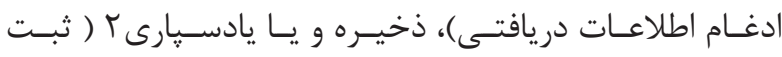

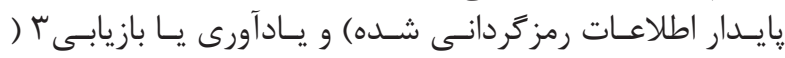
فراخوانـى اطلاعـات اندوختــه شـدهـ).

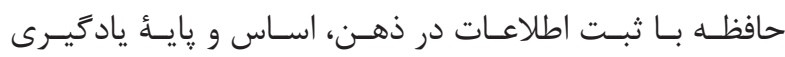

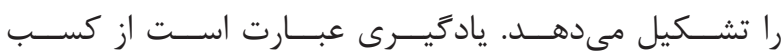

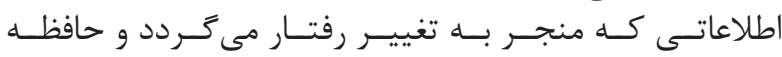

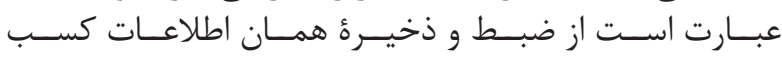

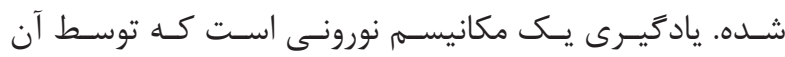

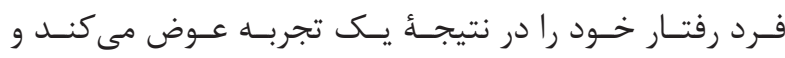

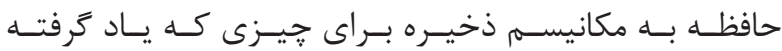

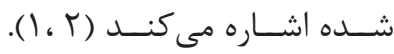

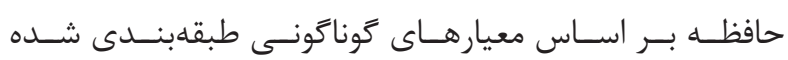

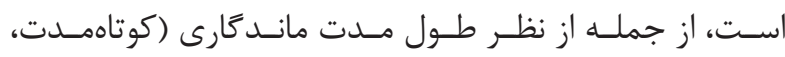

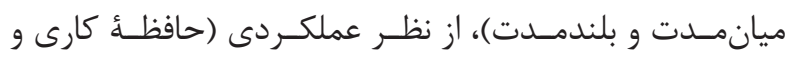

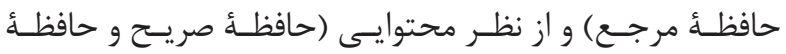

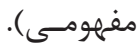

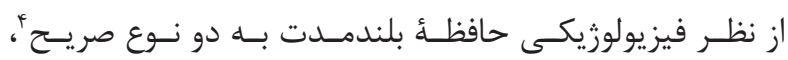

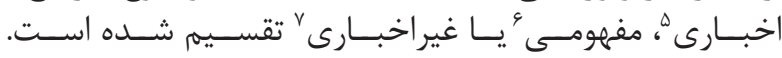

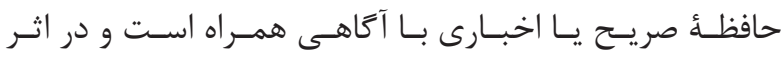

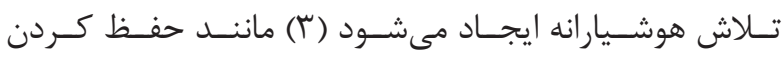

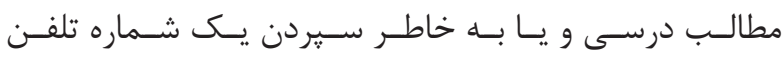

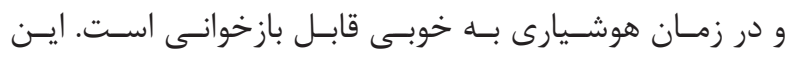

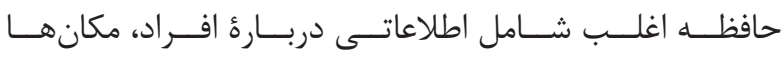

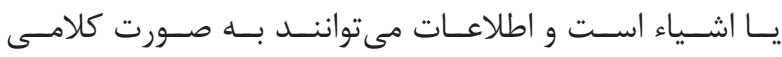

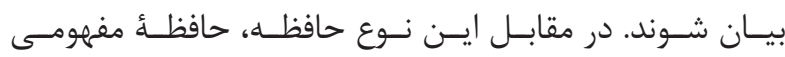

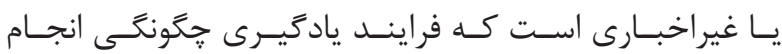

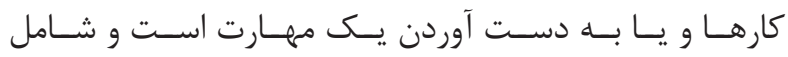

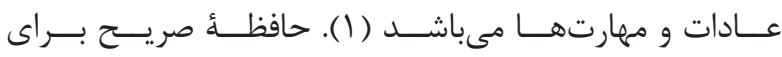

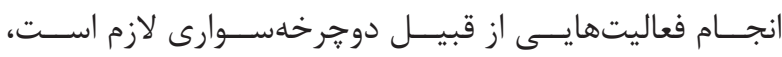

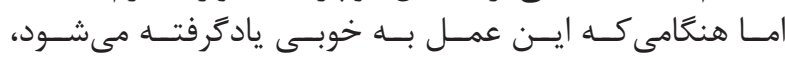

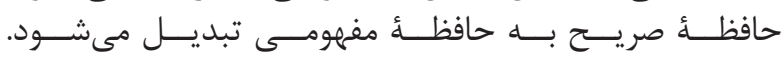

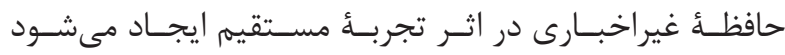

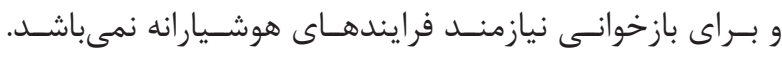

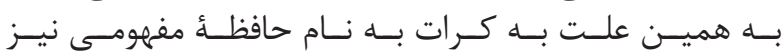

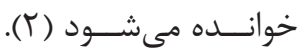

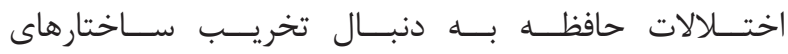

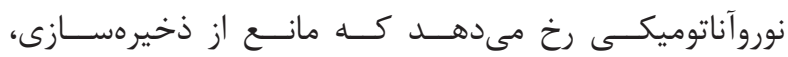

\footnotetext{
${ }^{10}$ Werniche-kosakoff syndrom

${ }^{11}$ Open field habituation

${ }^{12}$ Passive avoidance test

${ }^{13}$ Step-down inhibitory avoidance task

${ }^{14}$ Active avoidance test

${ }^{15}$ Morris water maze

168 -arms radial maze

${ }^{17}$ Novel object recognition test
} 
بلندشـدن روى دو يـاى عقبـى حالـت اكتشـاف ذاتـى جوند

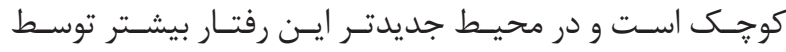

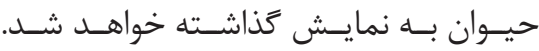

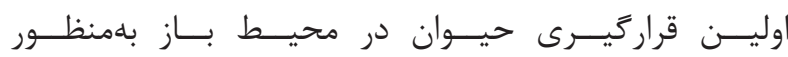

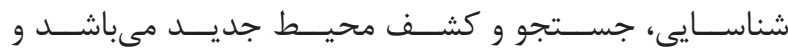

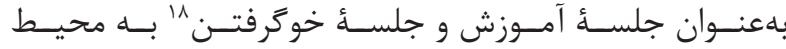

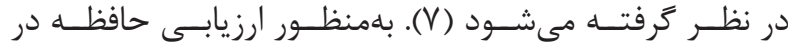

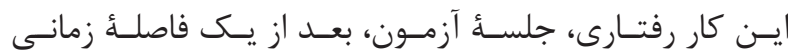

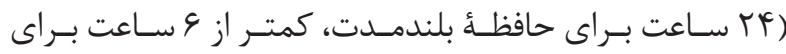

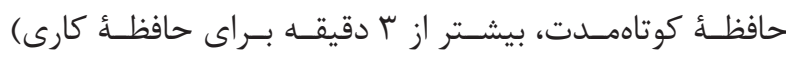

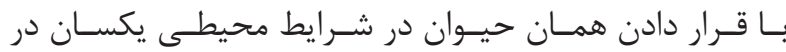

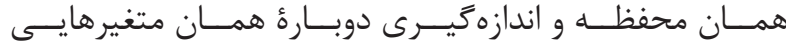

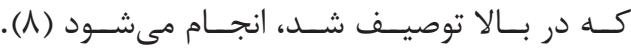

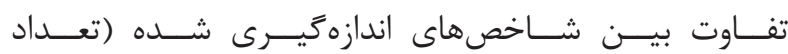

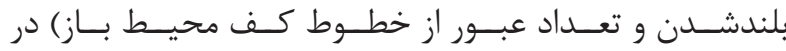

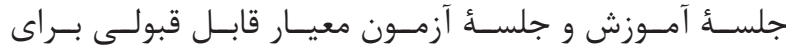

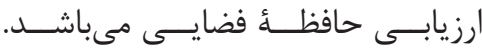

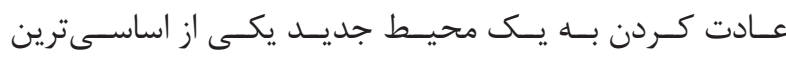

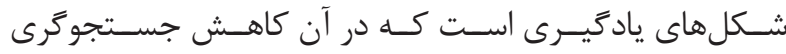

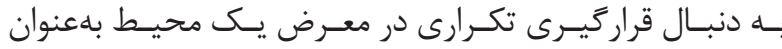

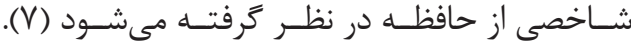

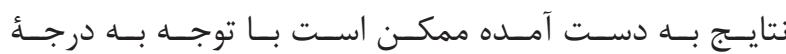

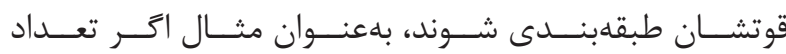

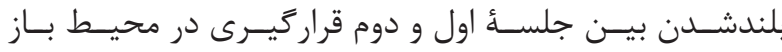

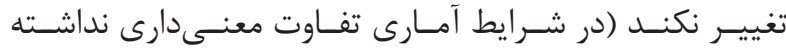

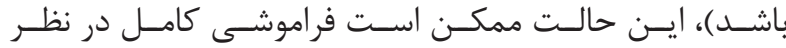

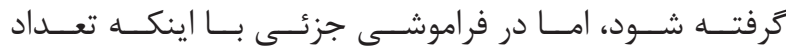

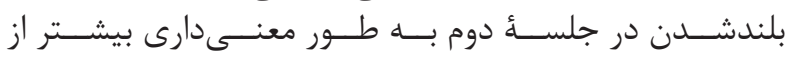

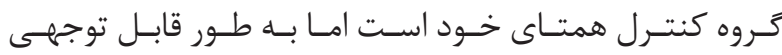

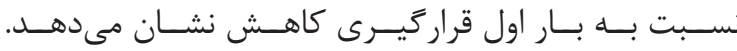

آزمون ماز Y (آ شكل

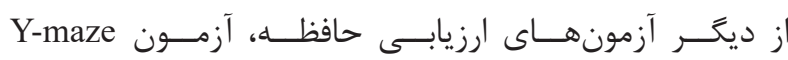

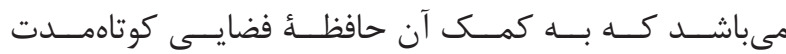

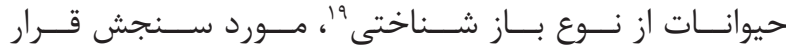

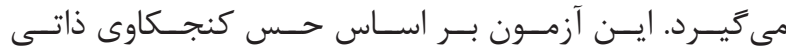

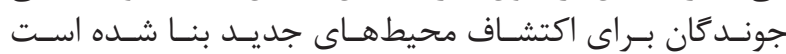

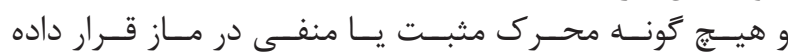

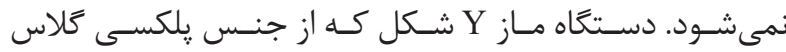

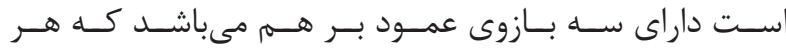

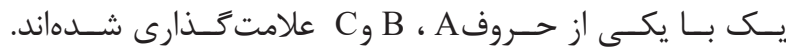

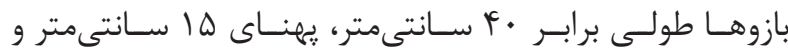

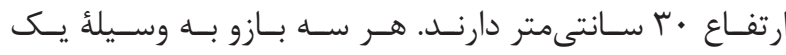

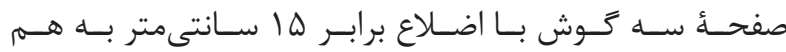

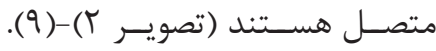

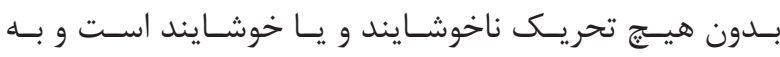

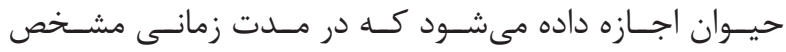

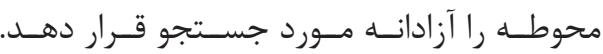

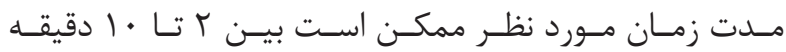

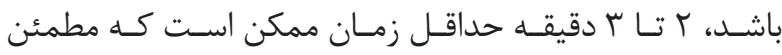

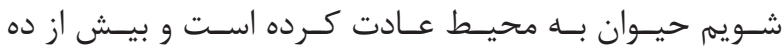

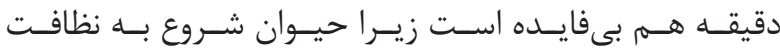

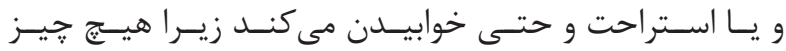

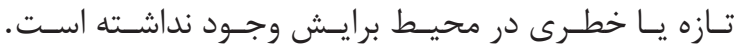

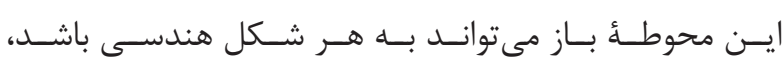

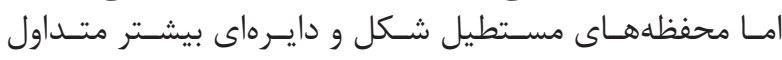

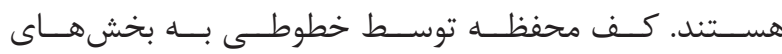

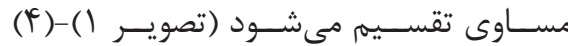

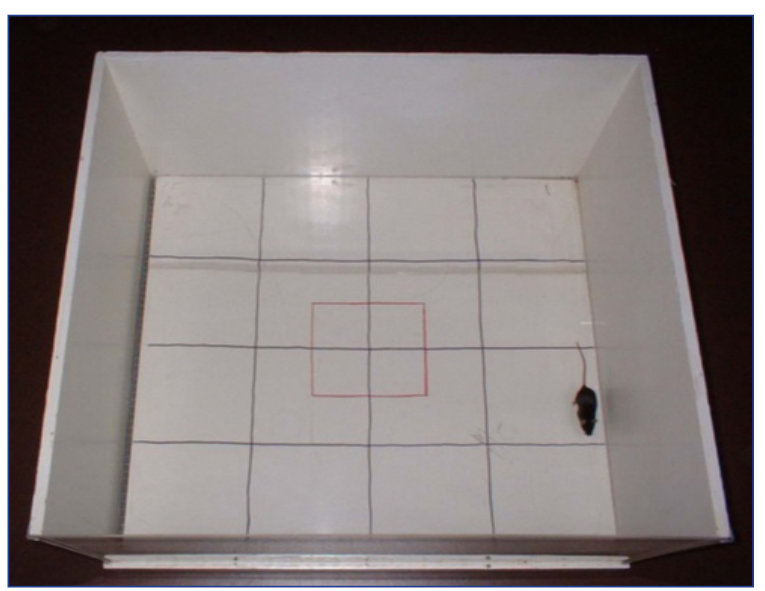

تصوير ا- آزمون عادت كردن به زمينة باز (\&).).

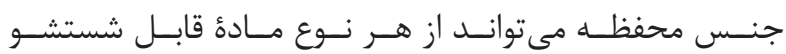

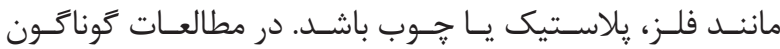

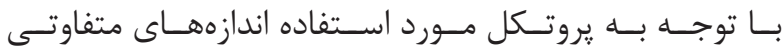

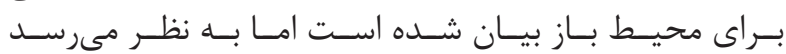

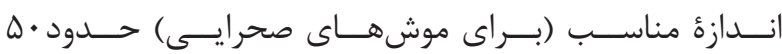

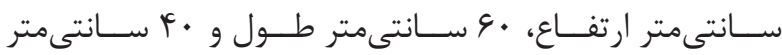

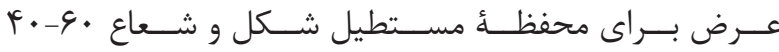

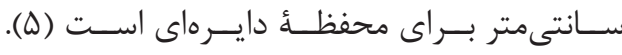

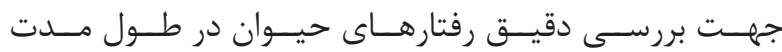

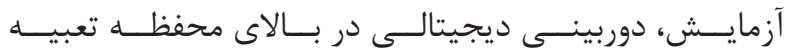

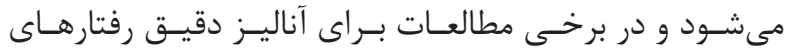

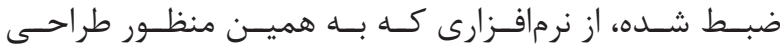

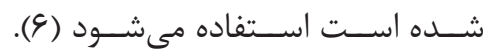

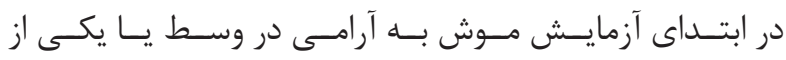

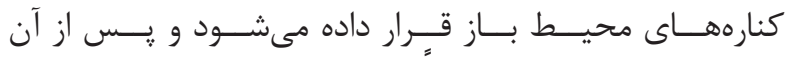

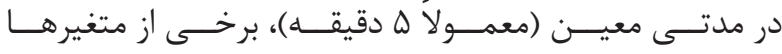

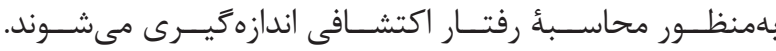

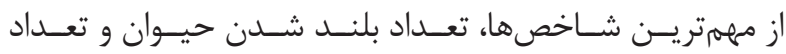

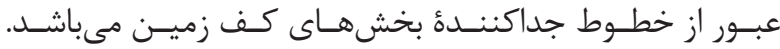




$$
\text { آزمون اجتنابى غيرفعال }
$$

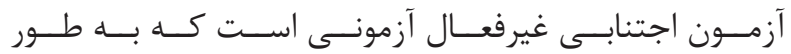

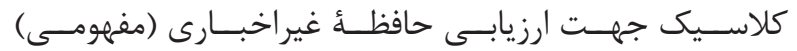

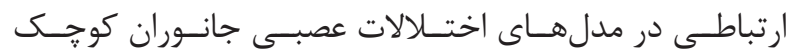

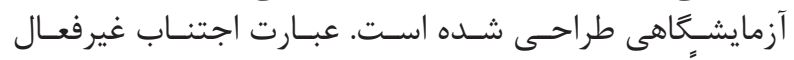

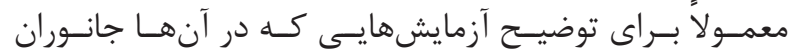

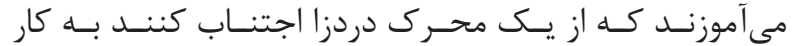

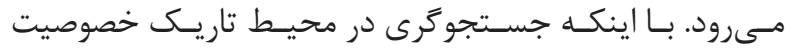

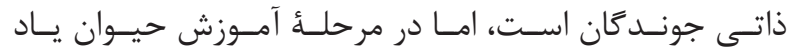

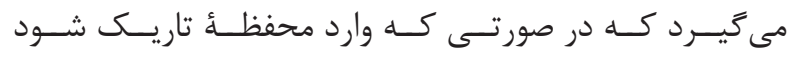

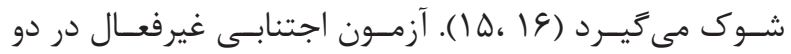

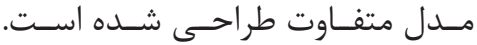

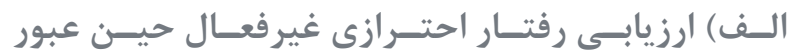

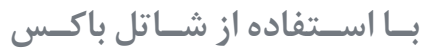

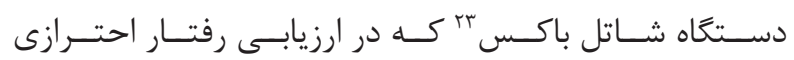

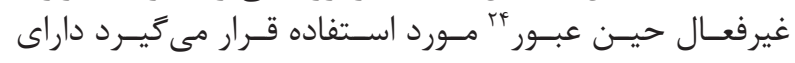

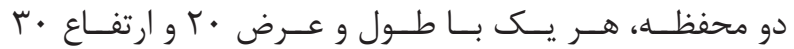

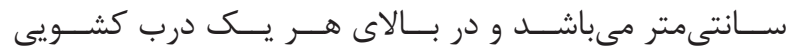

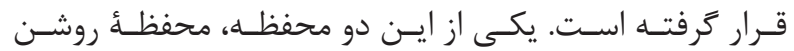

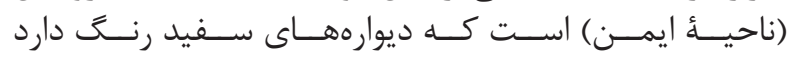

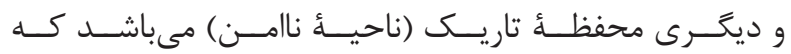

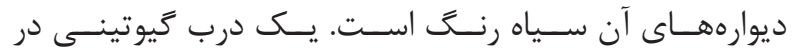

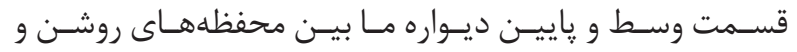

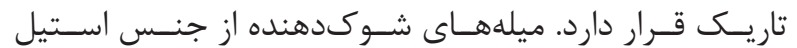

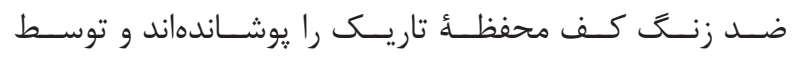

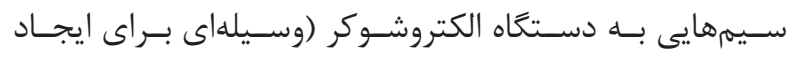

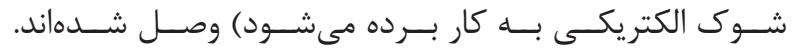

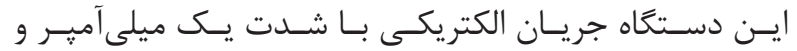

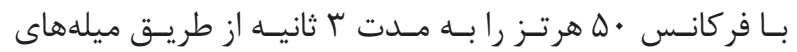

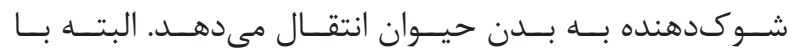

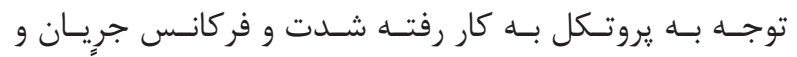

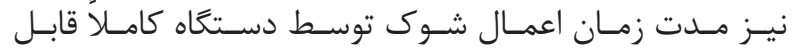

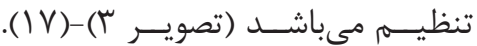

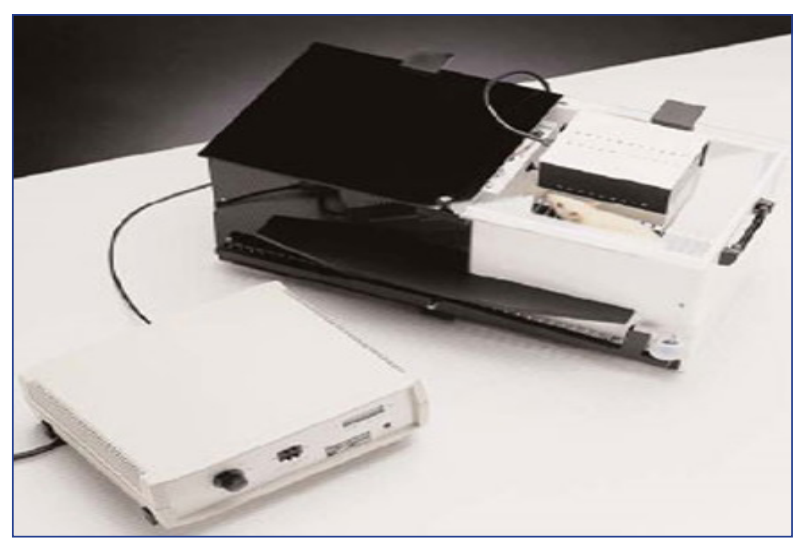

تصوير Y- دستكاه شاتل باكس (IV).

${ }^{20}$ Total number of entrance

${ }^{21}$ Actual alternation

${ }^{22}$ Alternation percentage

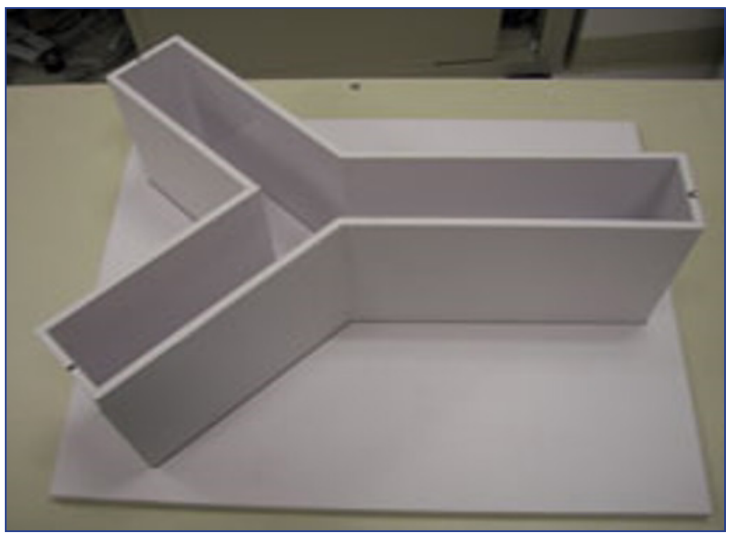

تصوير Y- دستخاه ماز Y شكل (9).

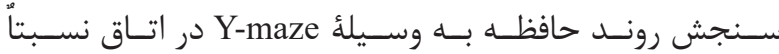

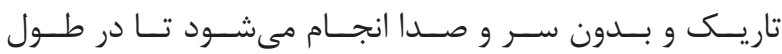

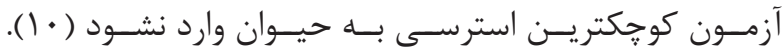

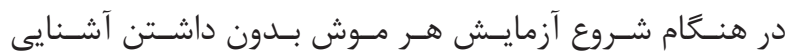

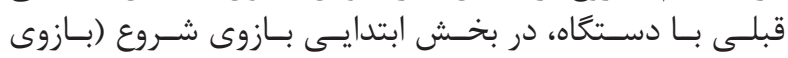

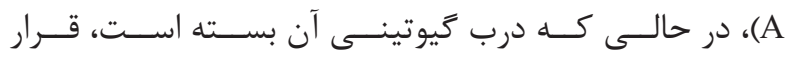

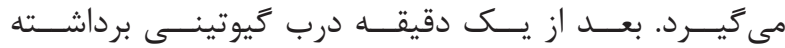

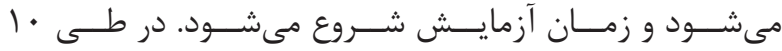

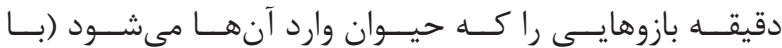

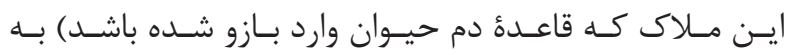

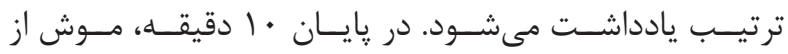

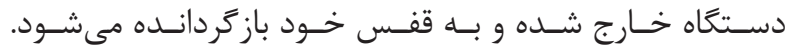

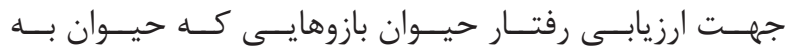

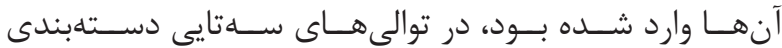

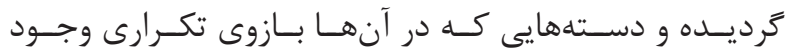

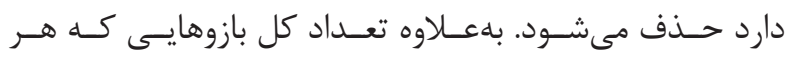

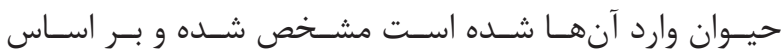

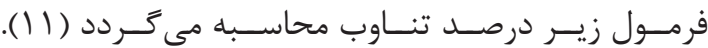

\section{$\left.\left\lceil\frac{\text { Actual alternation }}{(\text { Total number of entrance }}\right)^{-2}\right\rceil \times 100$}

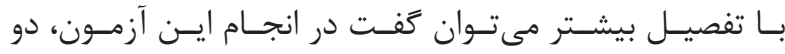

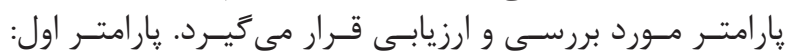

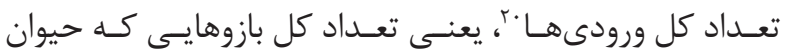

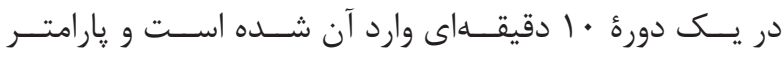

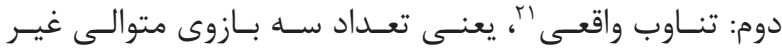

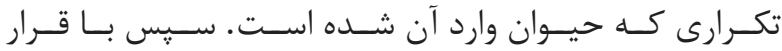

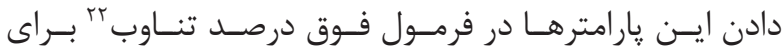

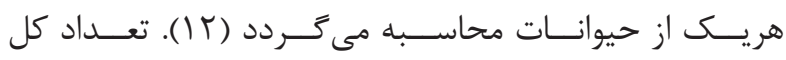

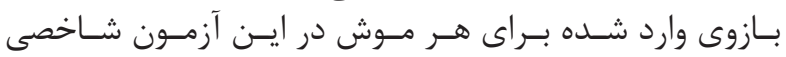

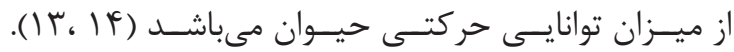

\footnotetext{
${ }^{23}$ Shuttle box

${ }^{24}$ Step through passive avoidance test
} 


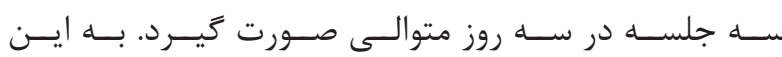

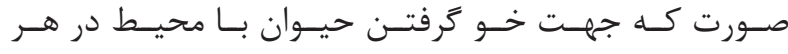

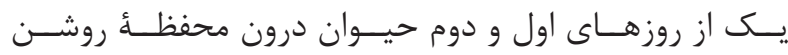

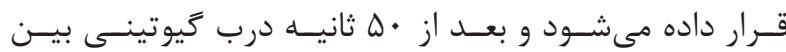

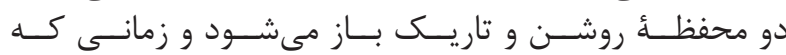

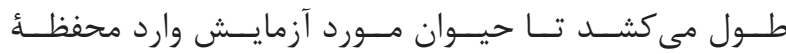

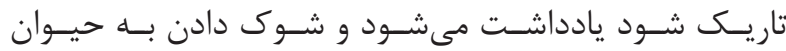

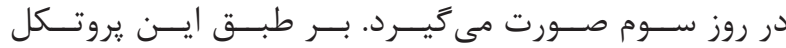

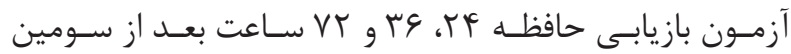

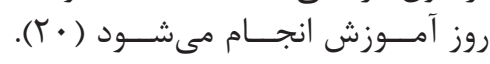

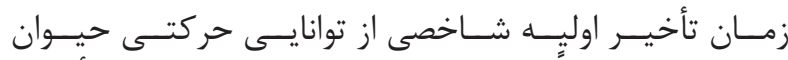

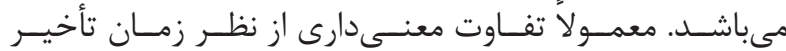

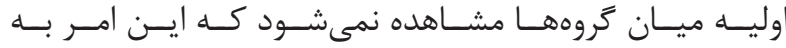

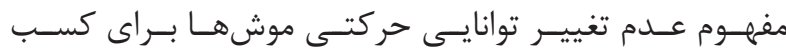

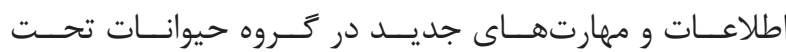

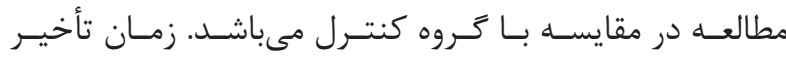

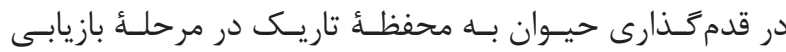

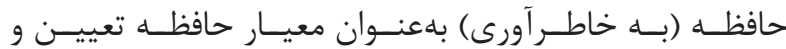

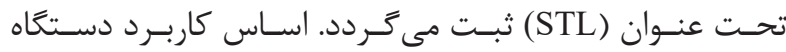

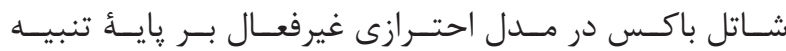

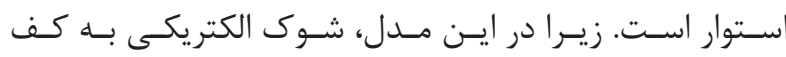

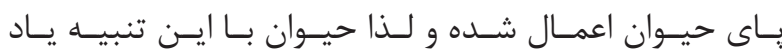

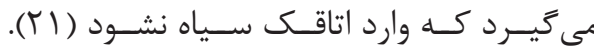

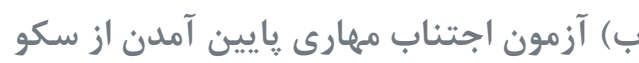

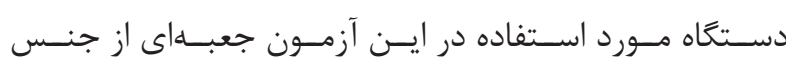

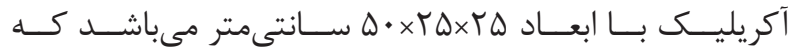

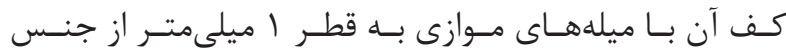

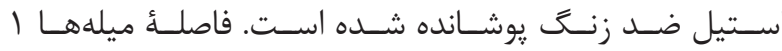

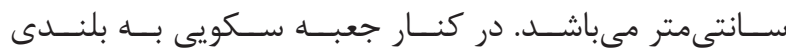

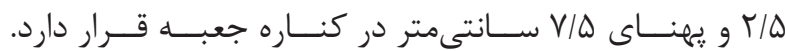

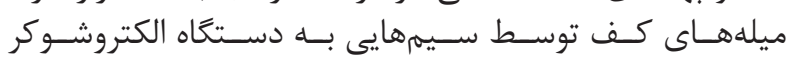

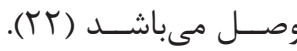

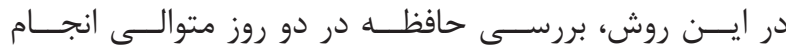

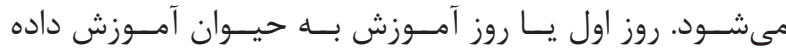

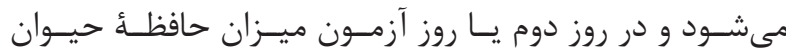

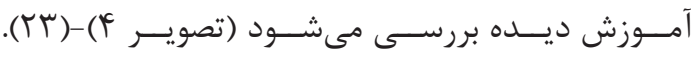

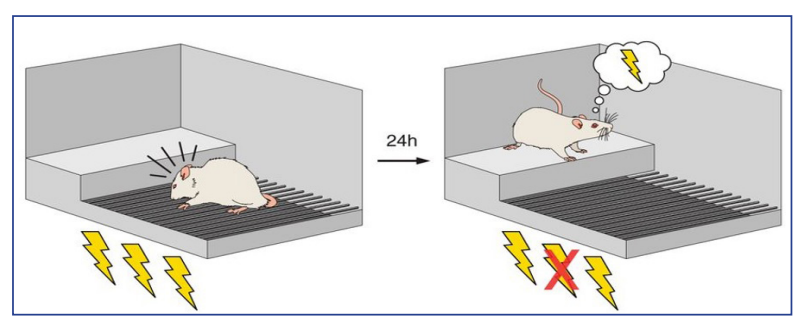

تصوير \&- آزمون اجتناب مهارى بايين آمدن از سكو (Tr).

${ }^{25}$ Training

${ }^{26}$ Initial latency

${ }^{27}$ Acquisition trial

$$
\text { روش بررسى رفتار احتر ازى غيرفعال }
$$

بررسى رفتـار احتـــازى غيرفعـال طـى دو روز متوالى انجـام

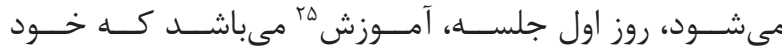

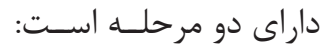

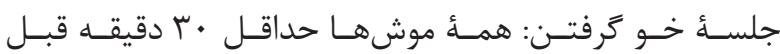

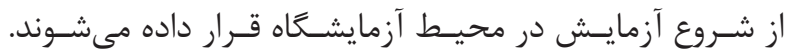

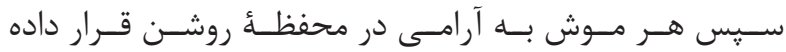

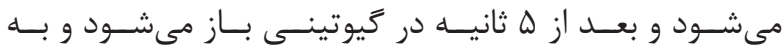

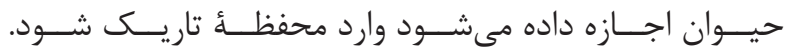

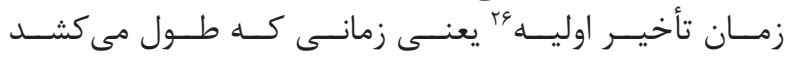

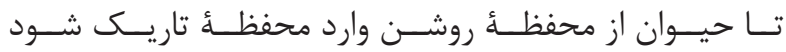

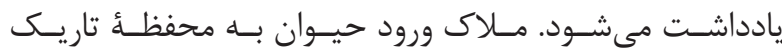

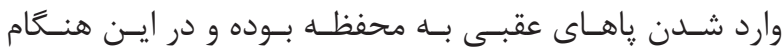

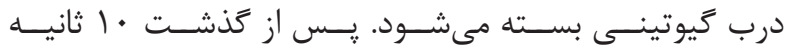

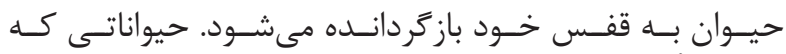

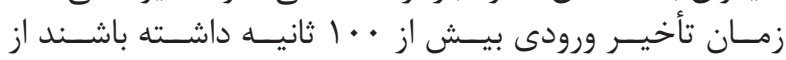

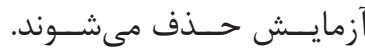

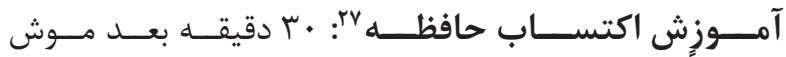

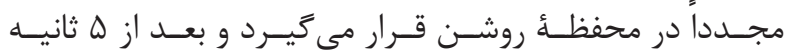

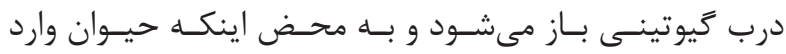

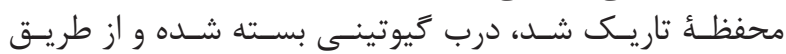

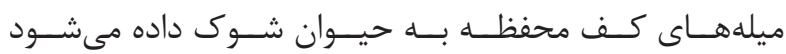

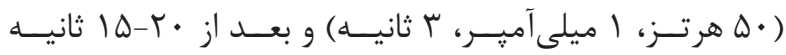

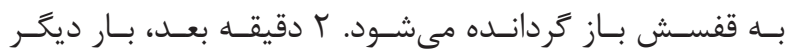

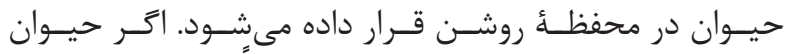

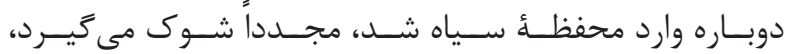

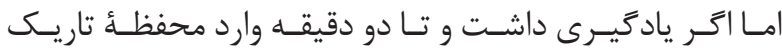

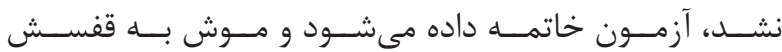

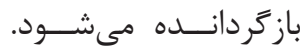
آزمون بازيابى حافظه

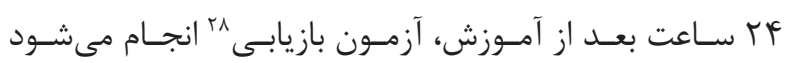

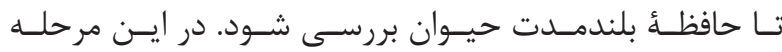

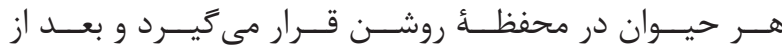

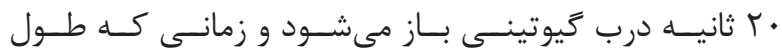

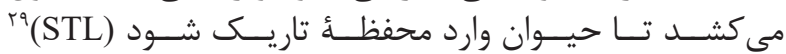

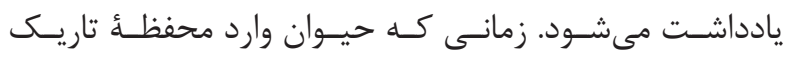

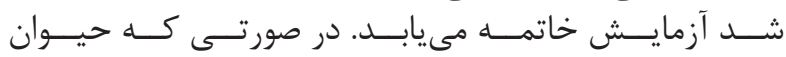

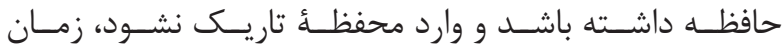

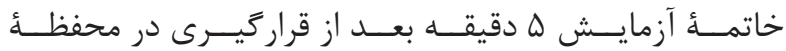

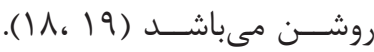

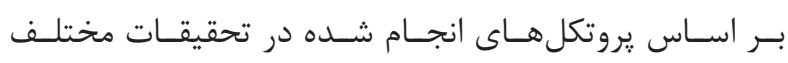

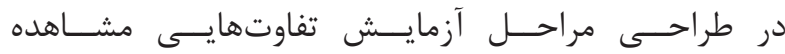

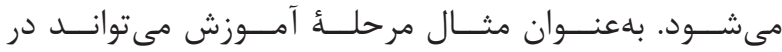

${ }^{28}$ Retrieval test

${ }^{29}$ Step-through latency 


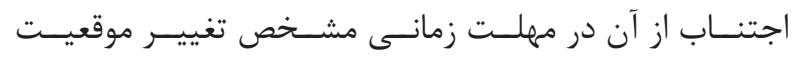

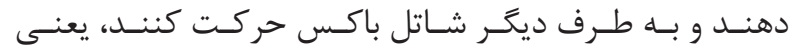

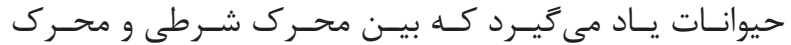

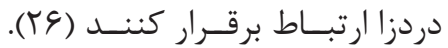

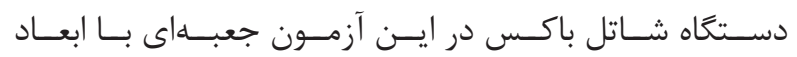

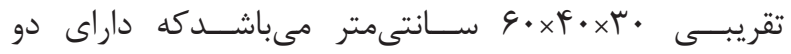

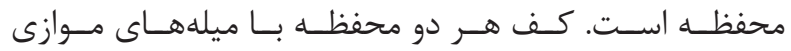

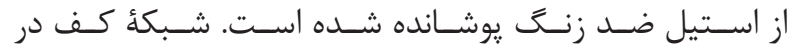

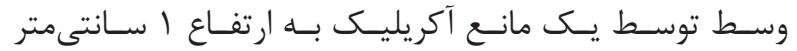

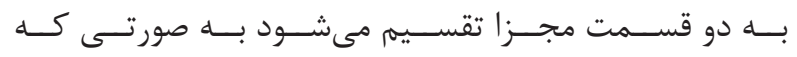

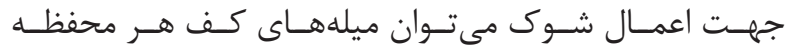

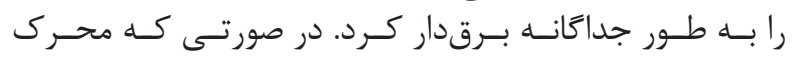

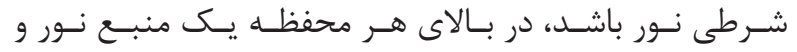

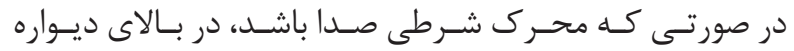

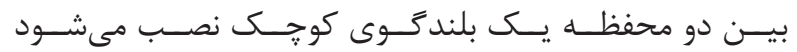

(تصويــر (Q) (TV)

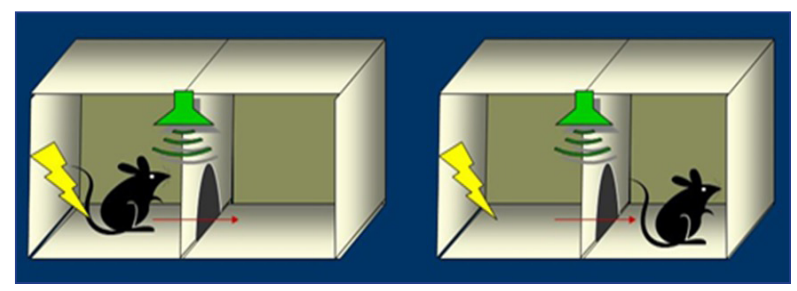

تصوير هـ آزمون اجتنابى فعال (TV).

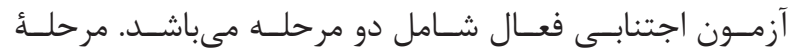

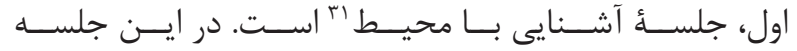

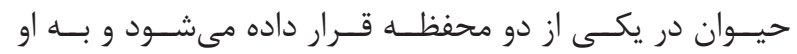

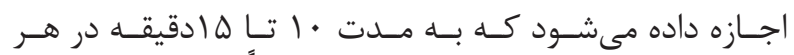

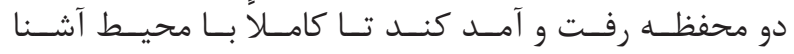

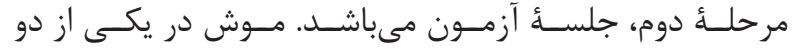

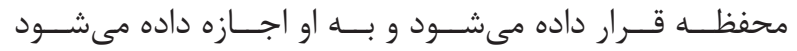

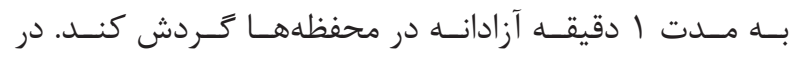

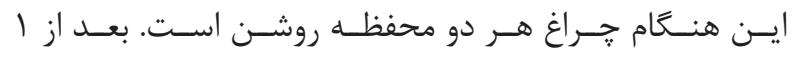

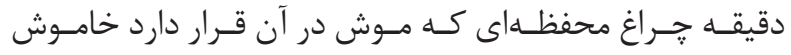

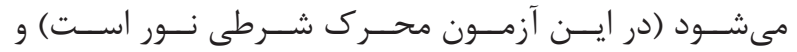

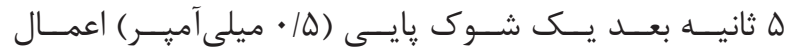

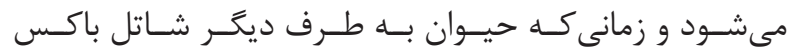

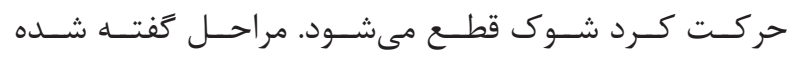

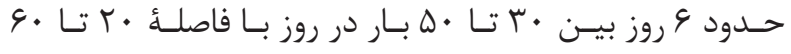

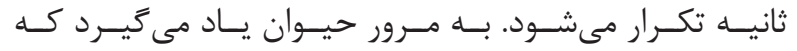

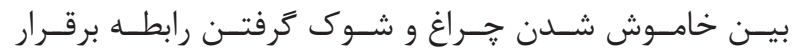

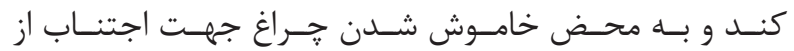

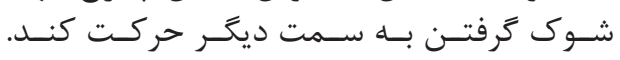

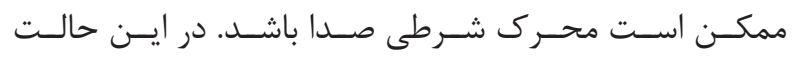

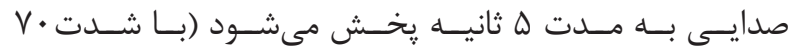

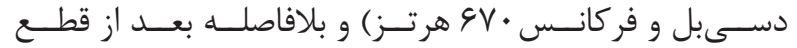

${ }^{30}$ Conditioned stimulus

${ }^{31}$ Habituation
مر حلة آموزش

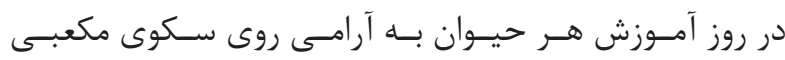

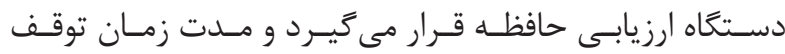

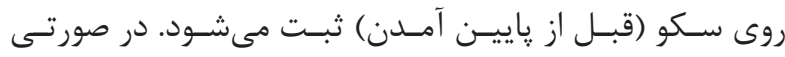

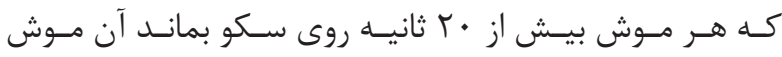

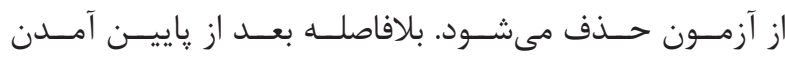

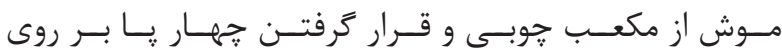

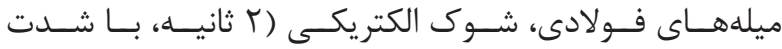

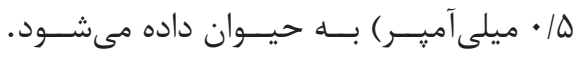
مر حلة آزمون يا بررسى حافظه آميه

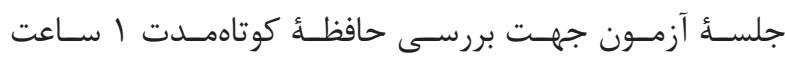

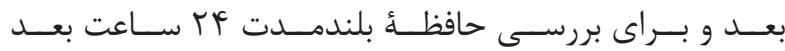

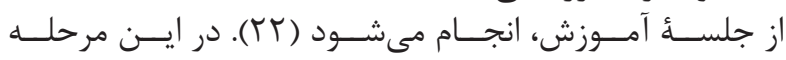

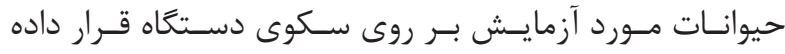

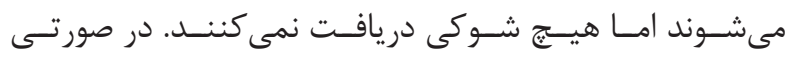

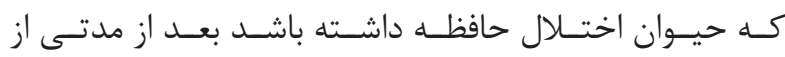

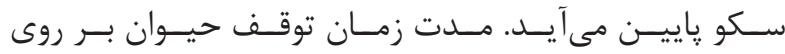

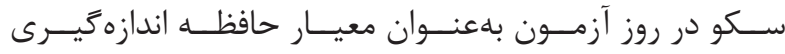

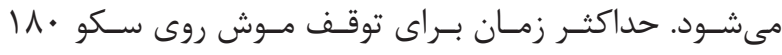

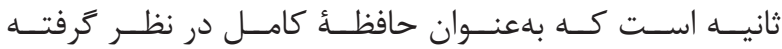

مىشـود (TF)

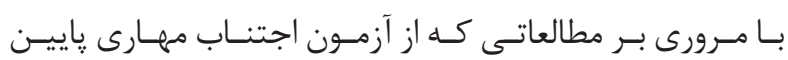

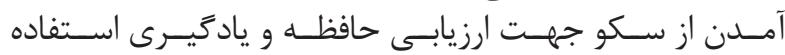

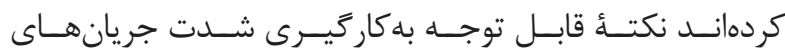

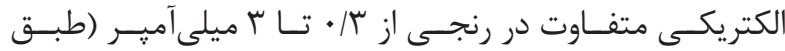

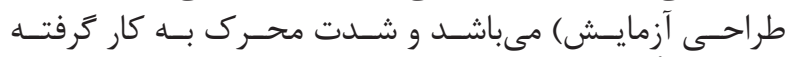

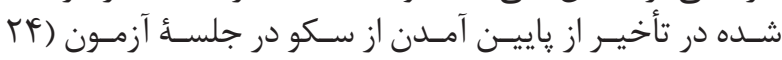

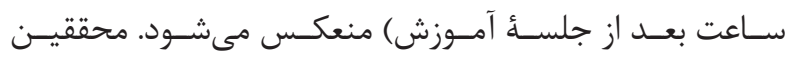

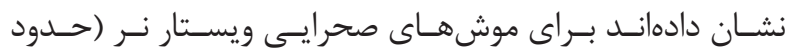

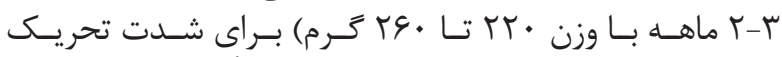

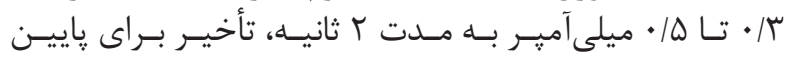

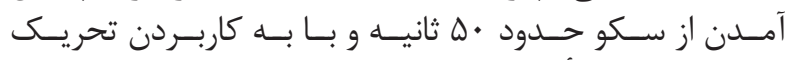

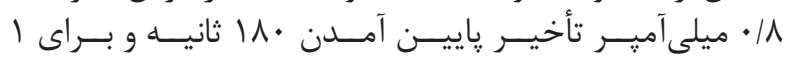

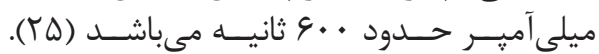

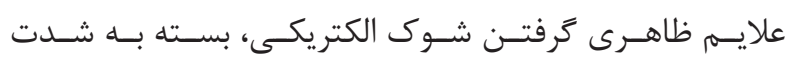

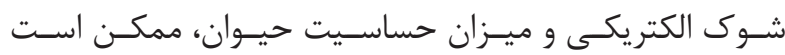

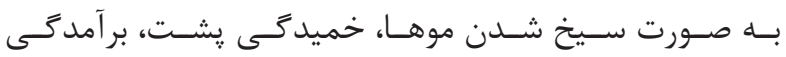

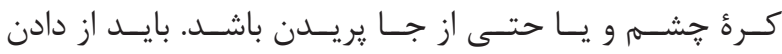

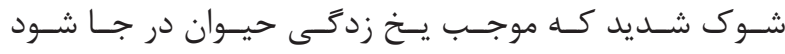

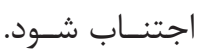
آزمون اجتنابى فعال

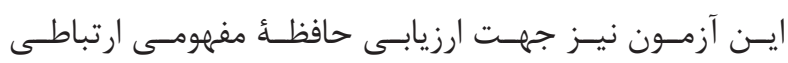

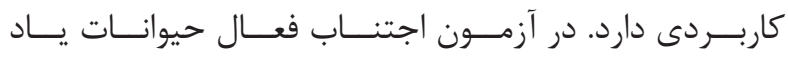

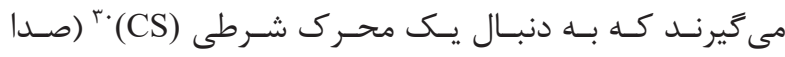

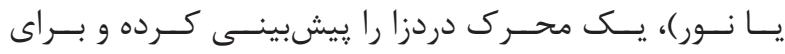




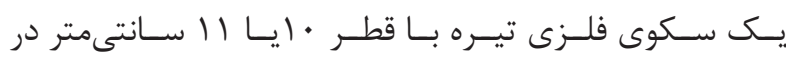

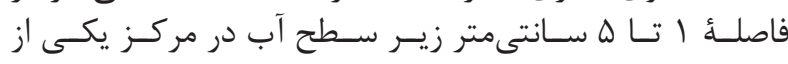

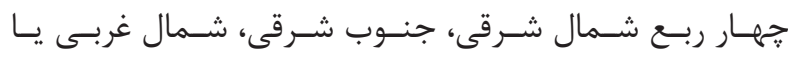

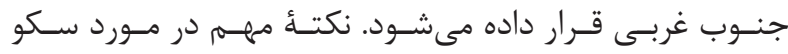

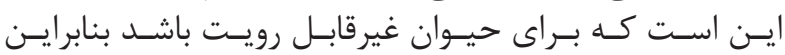

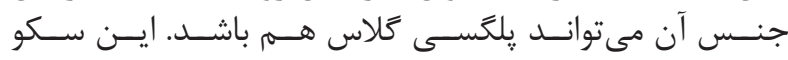

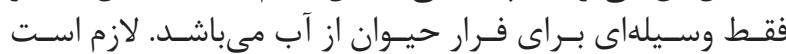

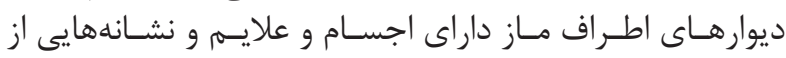

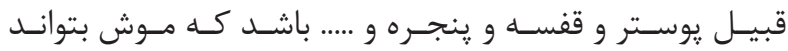

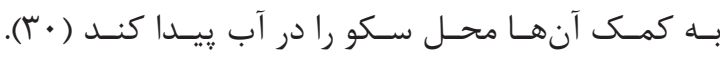

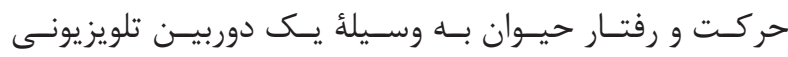

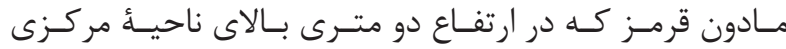

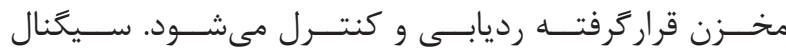

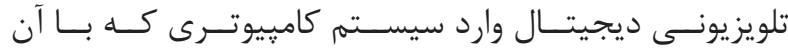

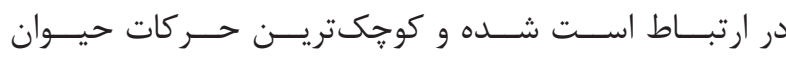

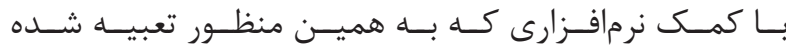

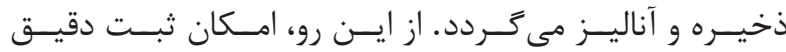

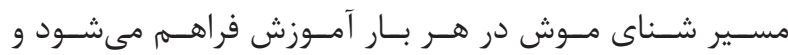

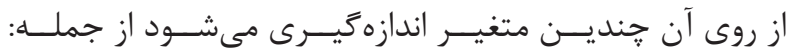

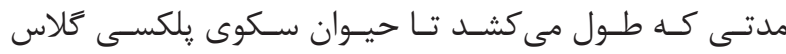

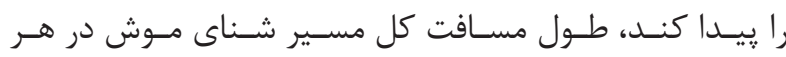

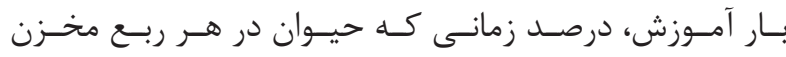

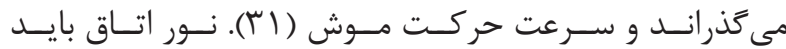

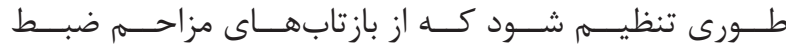

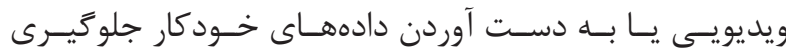

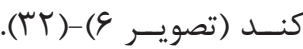

روش انجام آزمون ماز آبى موريس

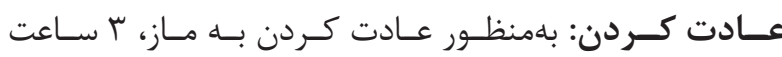

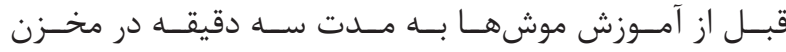

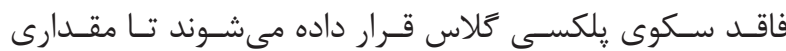
شـــا كنــــــ

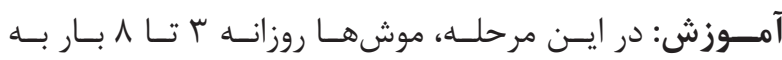

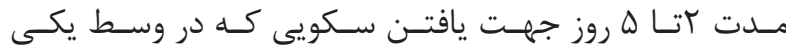

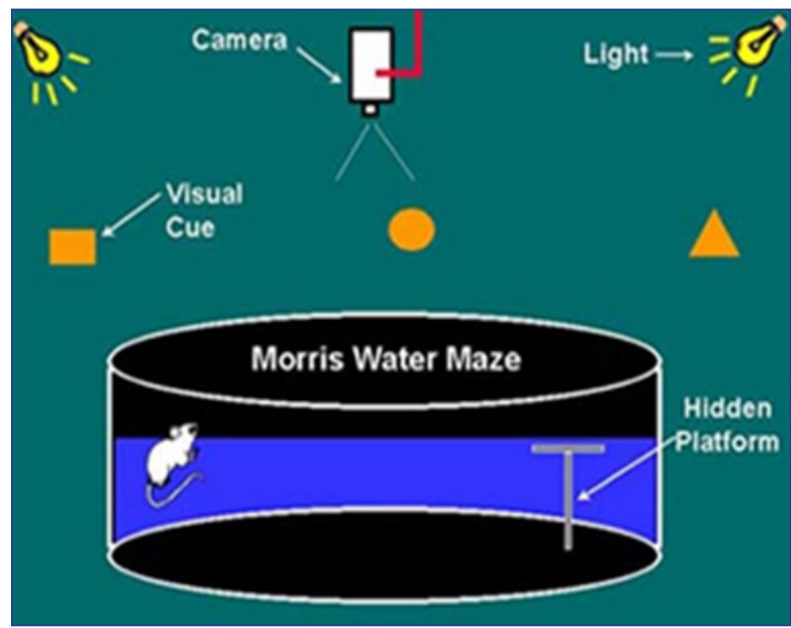

تصوير 9- ماز آبى موريس (YT).

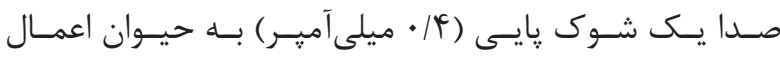

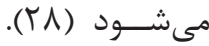

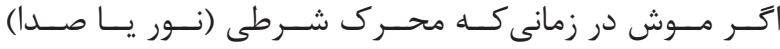

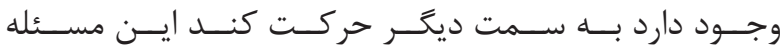

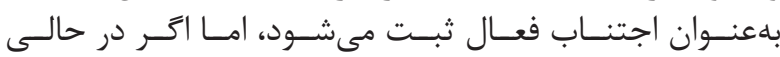

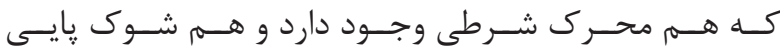

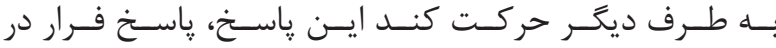

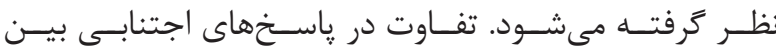

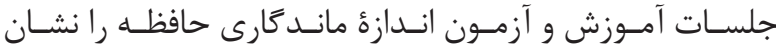

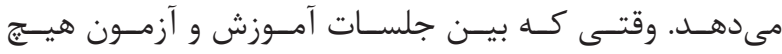

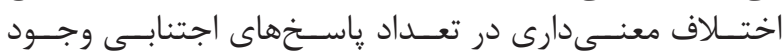

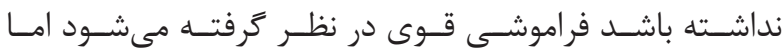

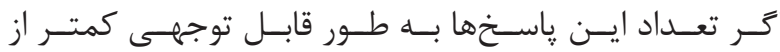

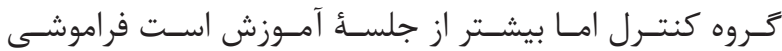

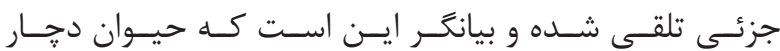

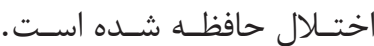

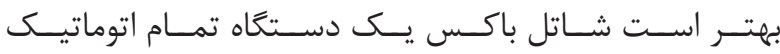

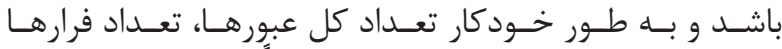

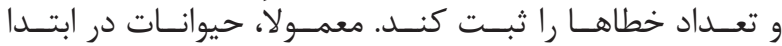

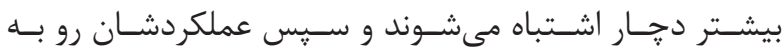

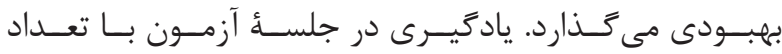

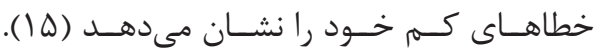

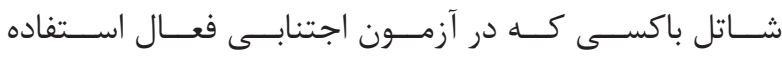

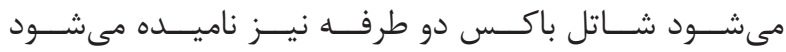

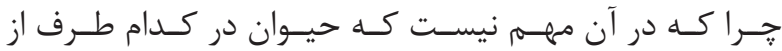

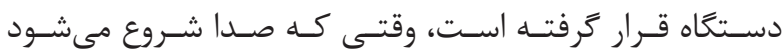

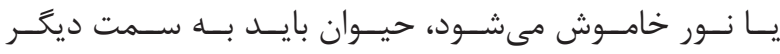

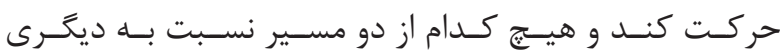

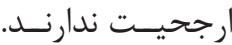

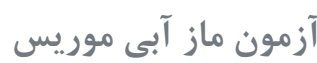

مــاز آبـى موريسـس يكـى از معمولتريسـن آزمونهــا در علـوم

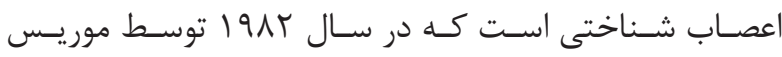

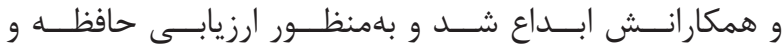

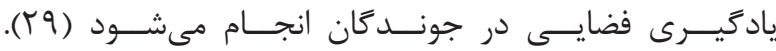

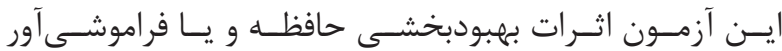

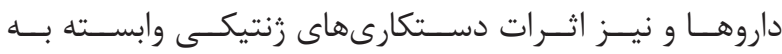

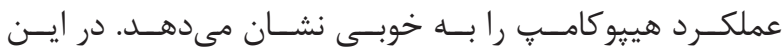

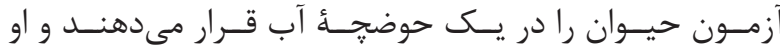

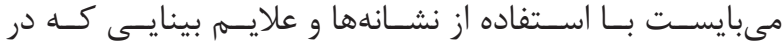

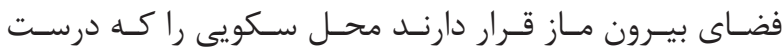

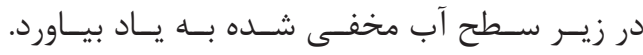

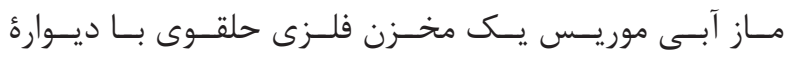

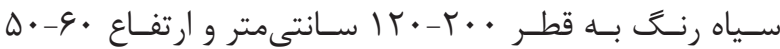

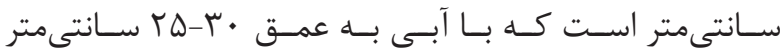

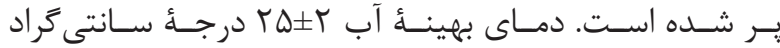
مىباشـــد. 


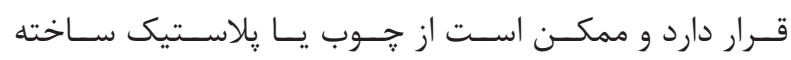

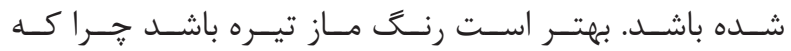

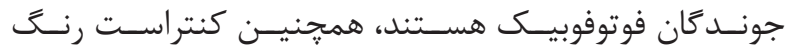

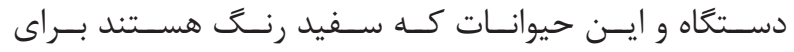

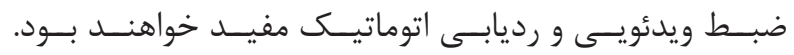

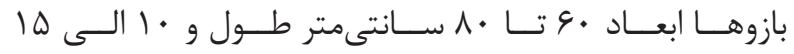

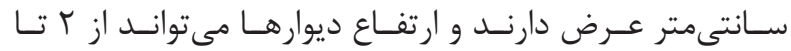

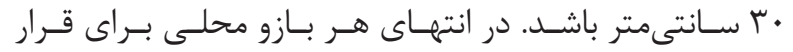

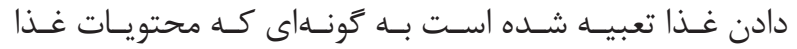

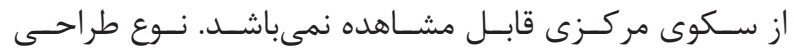

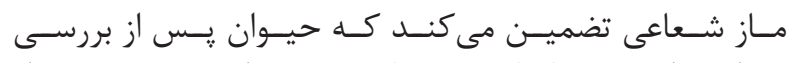

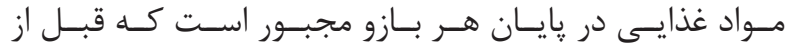

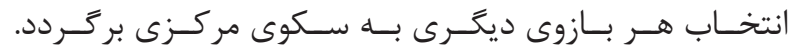

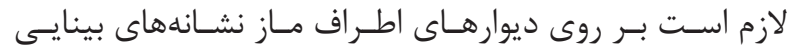

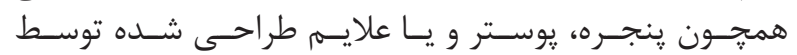

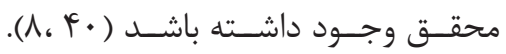

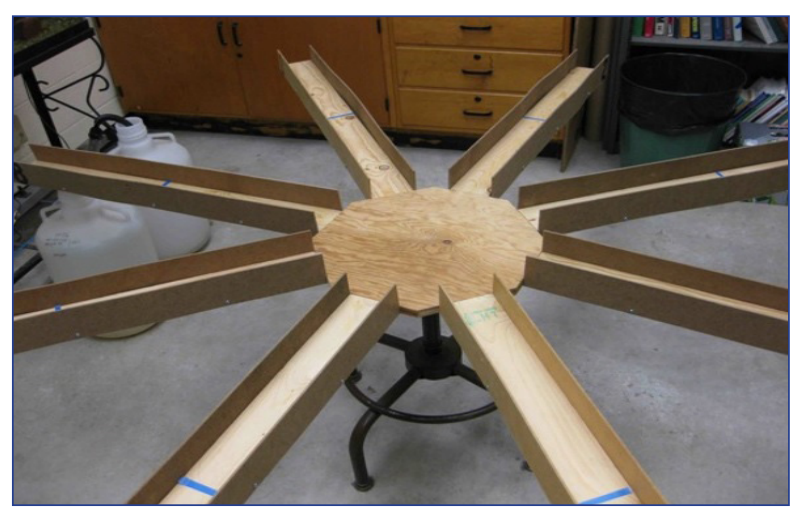

تصوير V- ماز شعاعى ^ بازويى (१"). روش انجام آزمون

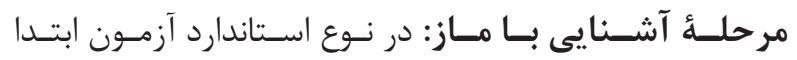

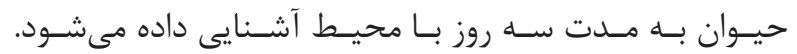

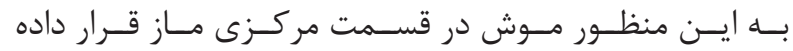

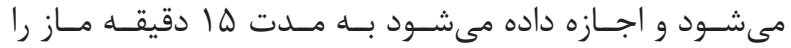

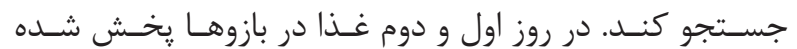

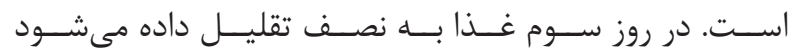

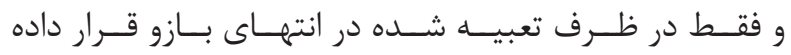

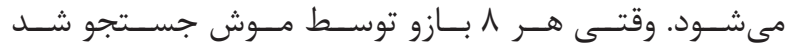

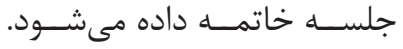

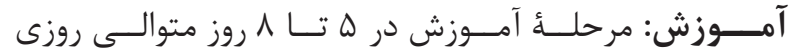

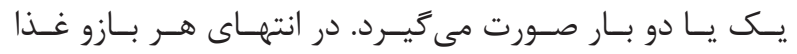

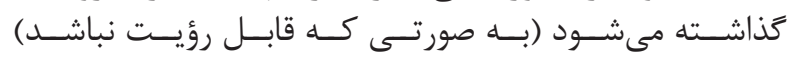

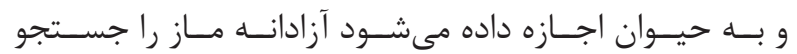

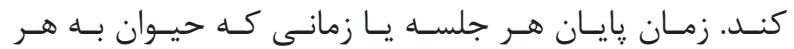

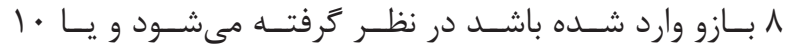

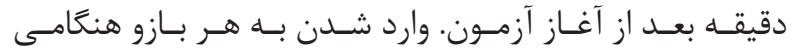

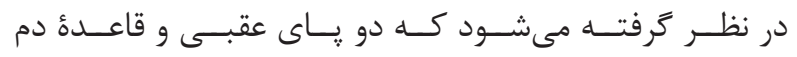

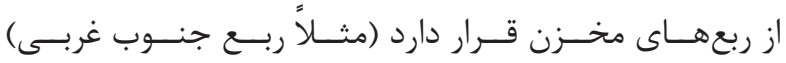

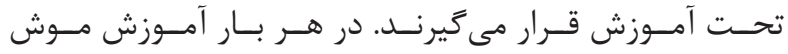

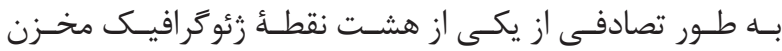

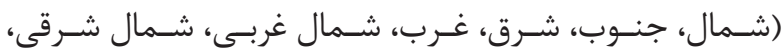

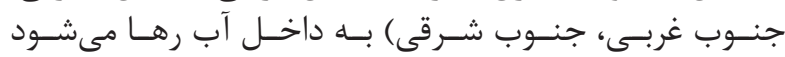

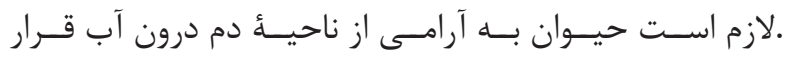

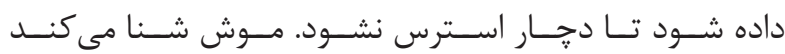

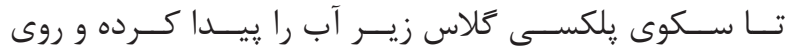

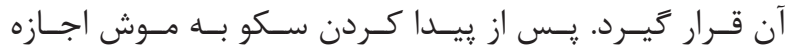

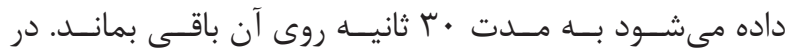

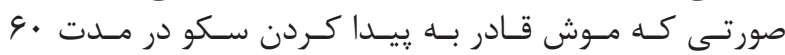

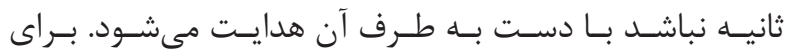

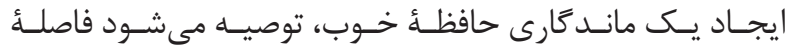

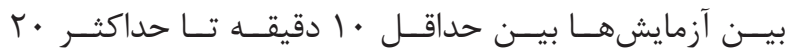

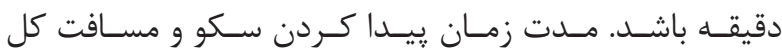

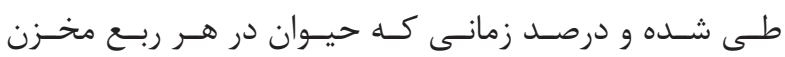

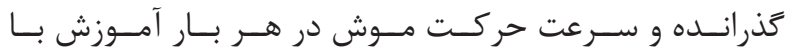

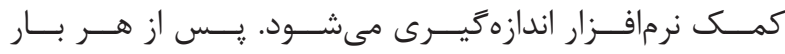

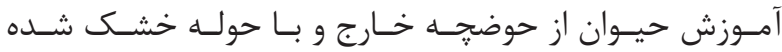

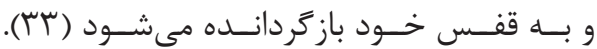

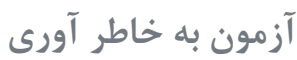

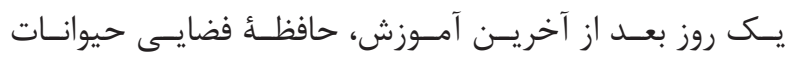

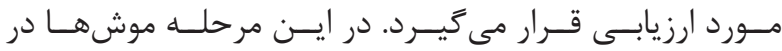

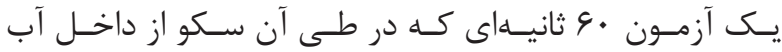

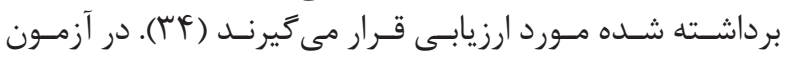

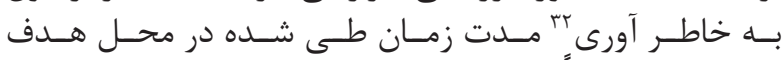

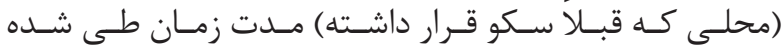

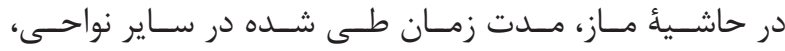

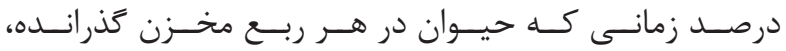

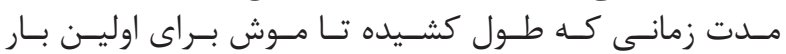

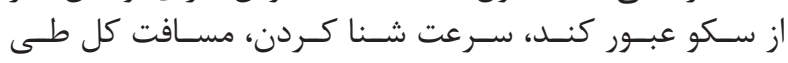

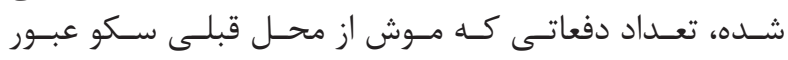

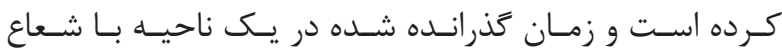

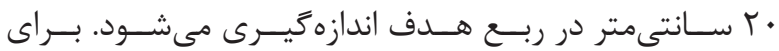

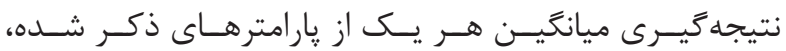

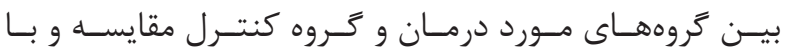

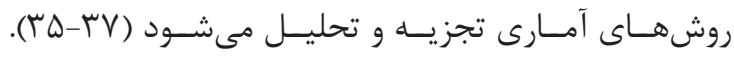

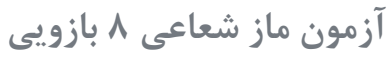

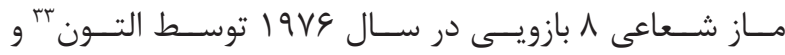

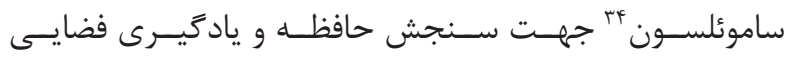

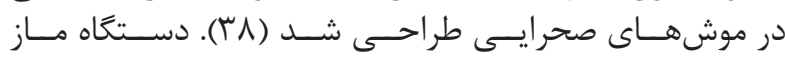

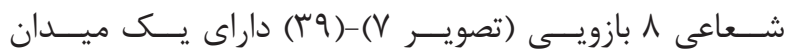

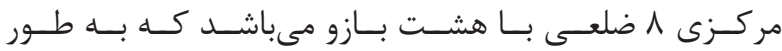

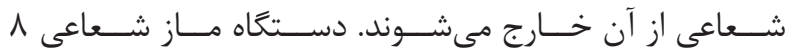

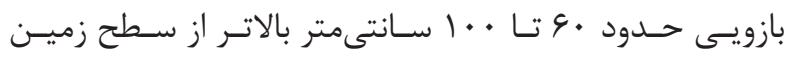

${ }^{32}$ Probe trial

${ }^{33}$ Olton

${ }^{34}$ Samuelson 
آزمون تشخيص شئ جديد

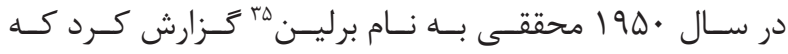

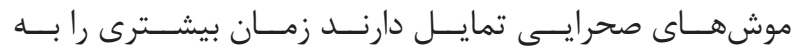

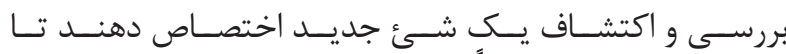

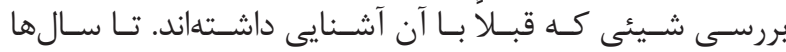

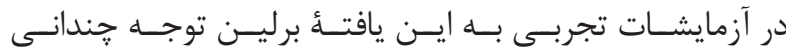

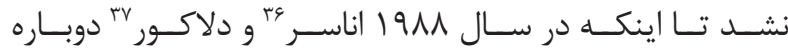

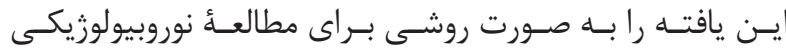

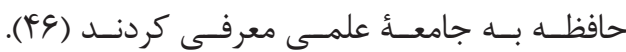

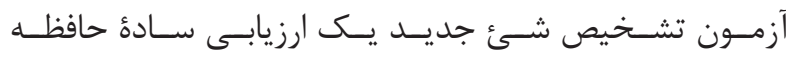

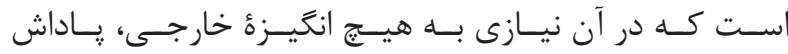

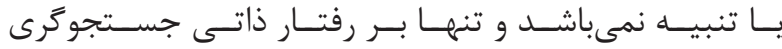

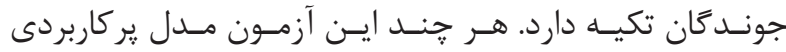

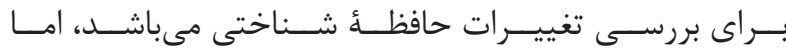

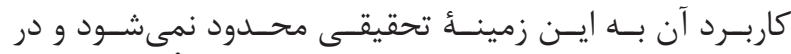

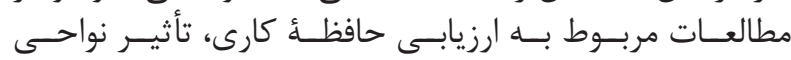

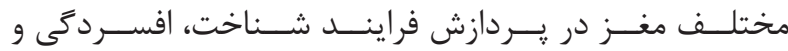

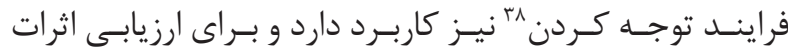

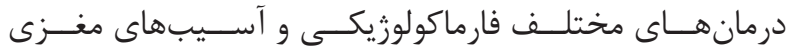

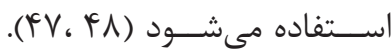

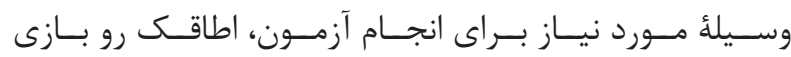

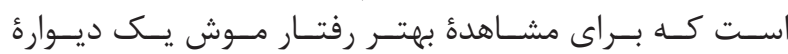

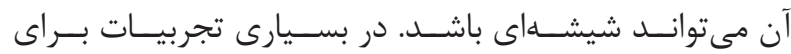

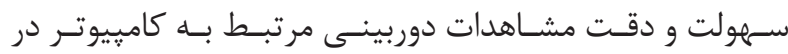

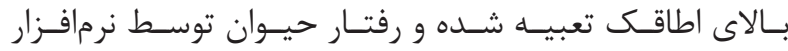

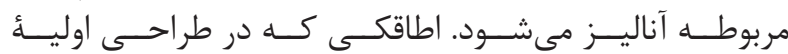

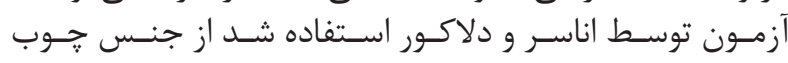

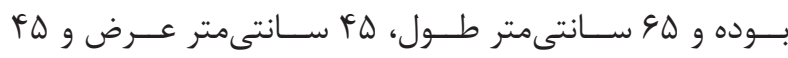

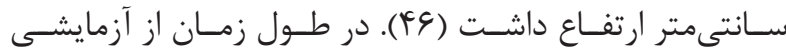

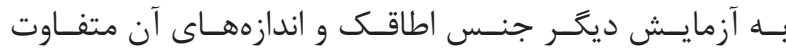

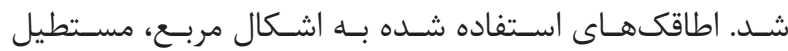

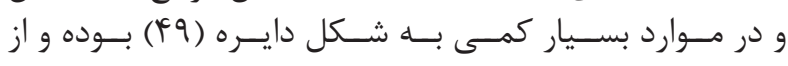

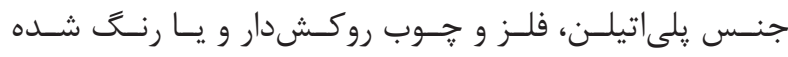

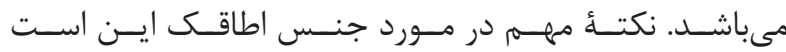

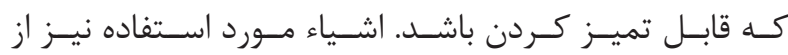

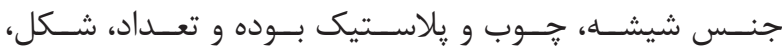

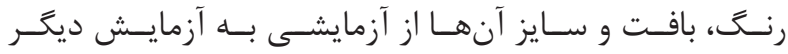

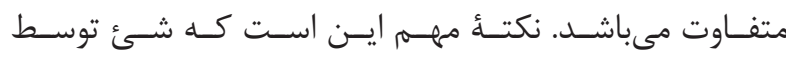

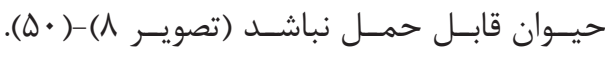

روش انجام آزمون

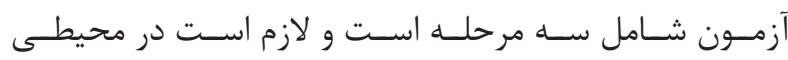

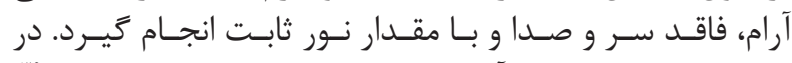

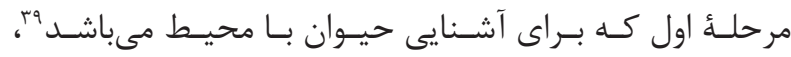

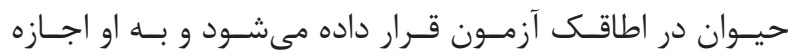

${ }^{35}$ Berlyne

${ }^{36}$ Ennaceur

${ }^{37}$ Delacour

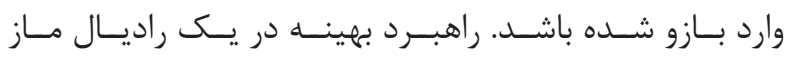

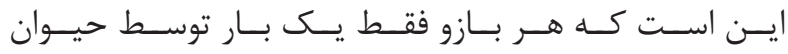

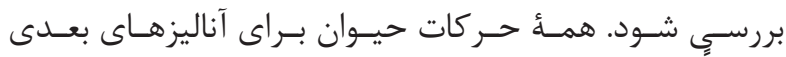

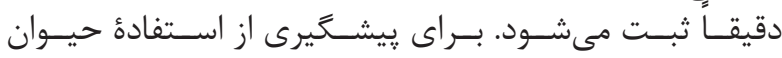

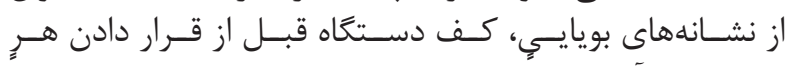

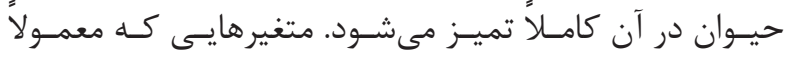

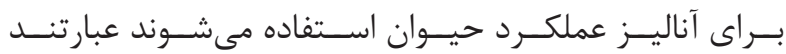

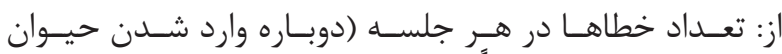

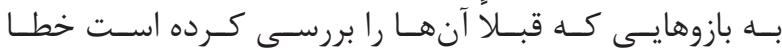

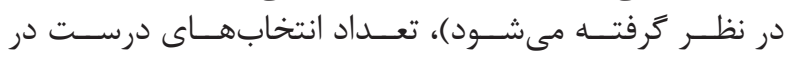

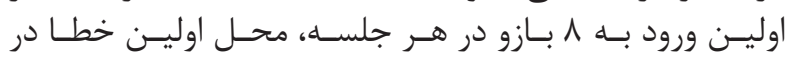

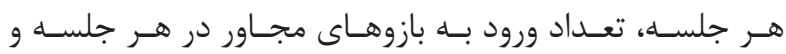

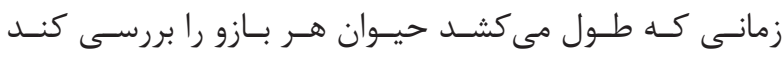

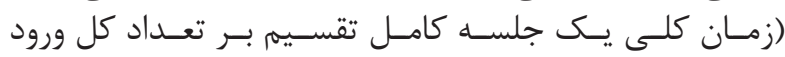

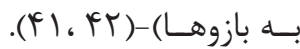

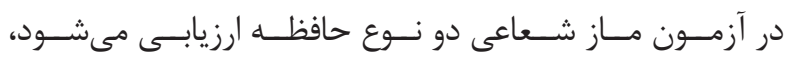

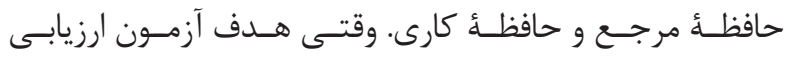

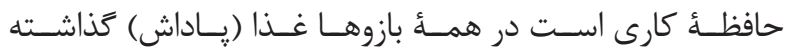

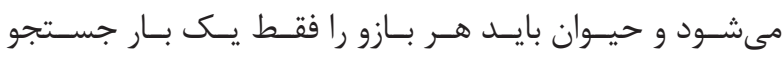

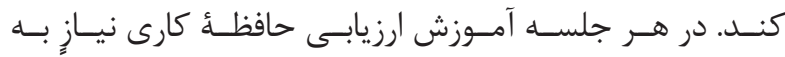

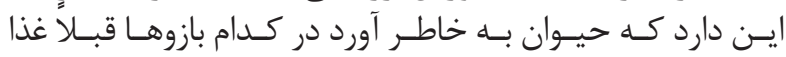

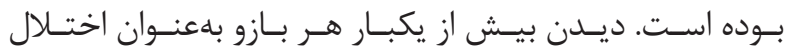

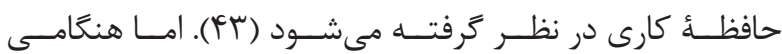

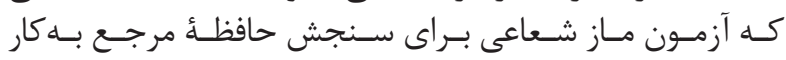

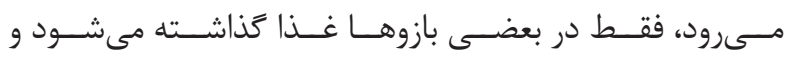

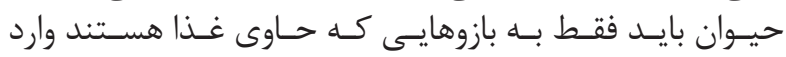

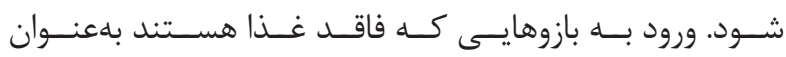

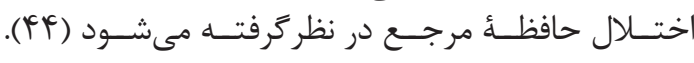

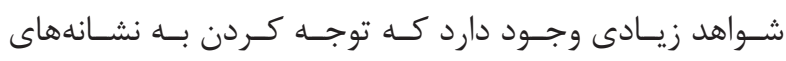

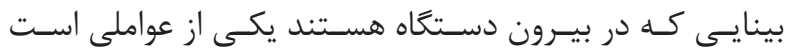

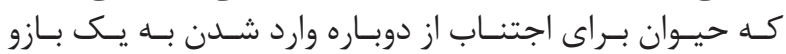

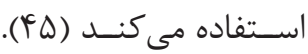

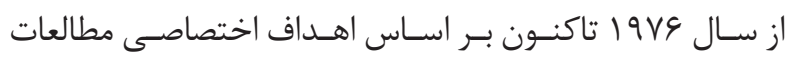

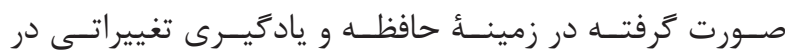

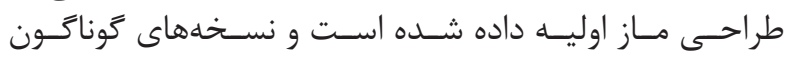

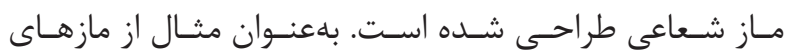

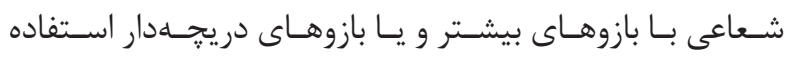

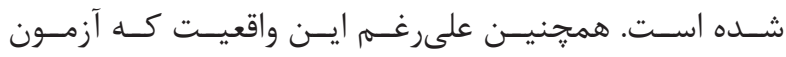

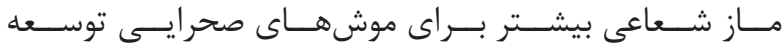

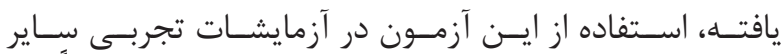

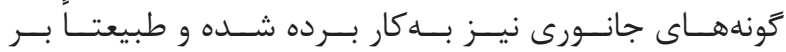

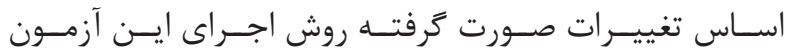

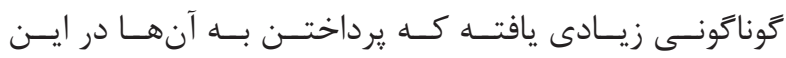

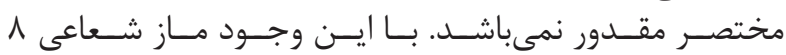

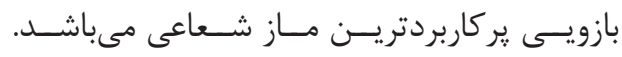

\footnotetext{
${ }^{38}$ Attention
}

${ }^{39}$ Habituation 


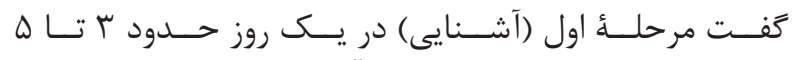

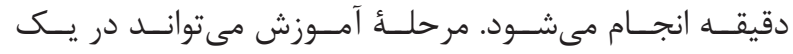

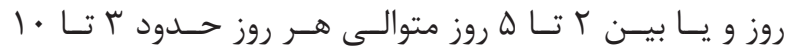

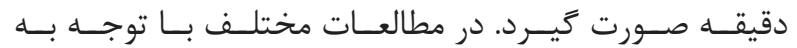

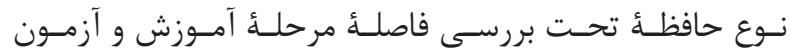

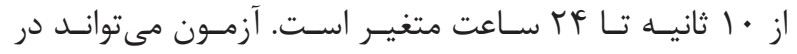

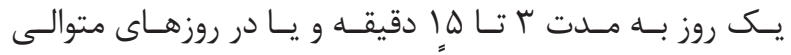

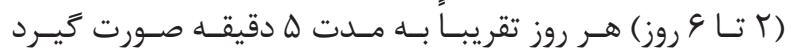

نتيجه

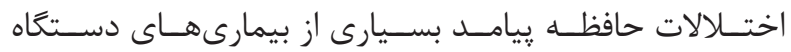

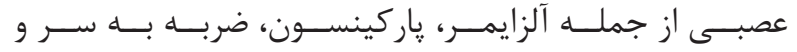

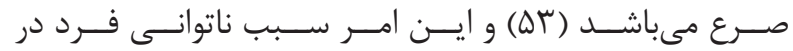

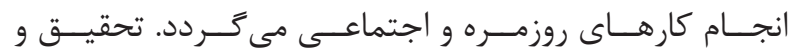

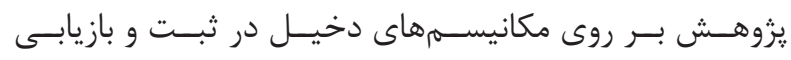

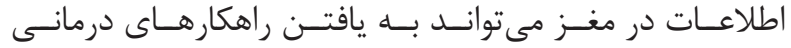

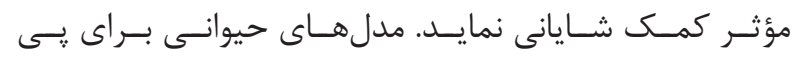

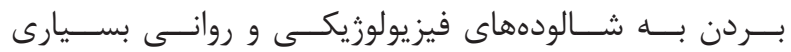

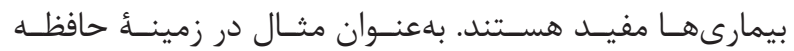

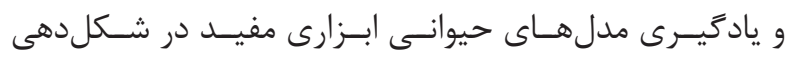

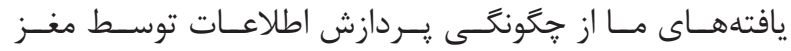

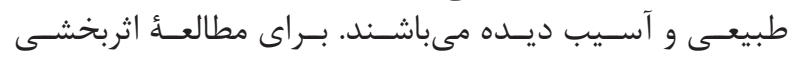

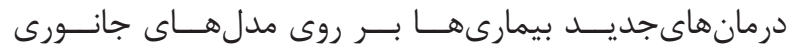

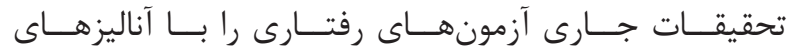

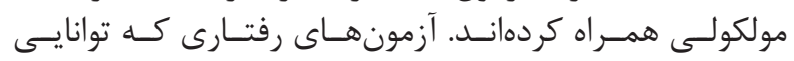

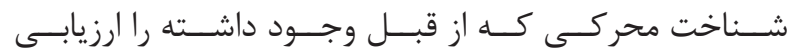

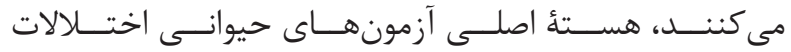

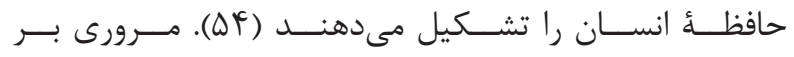

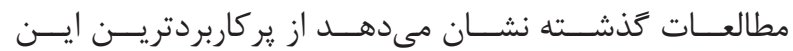

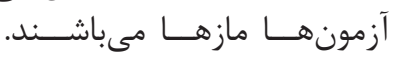

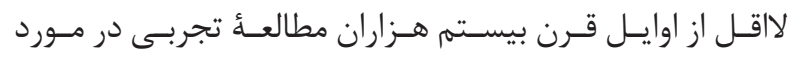

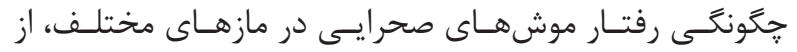

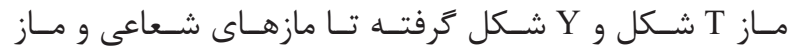

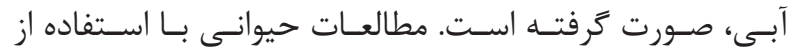

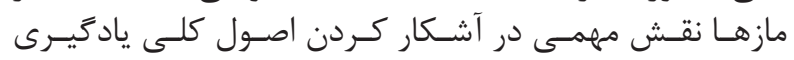

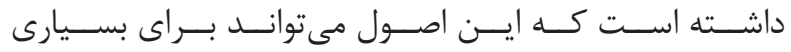

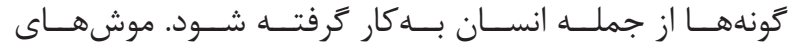

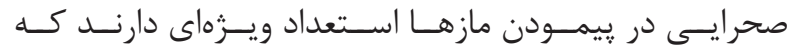

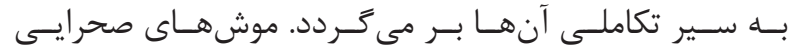

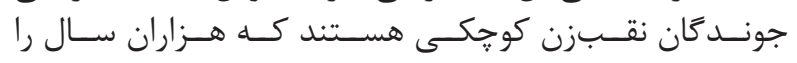

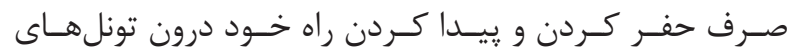

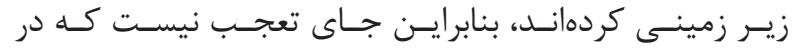

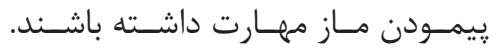

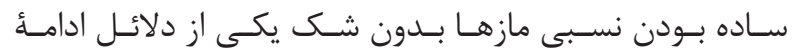

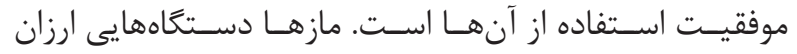

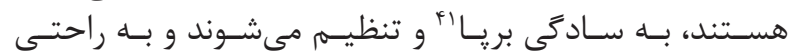

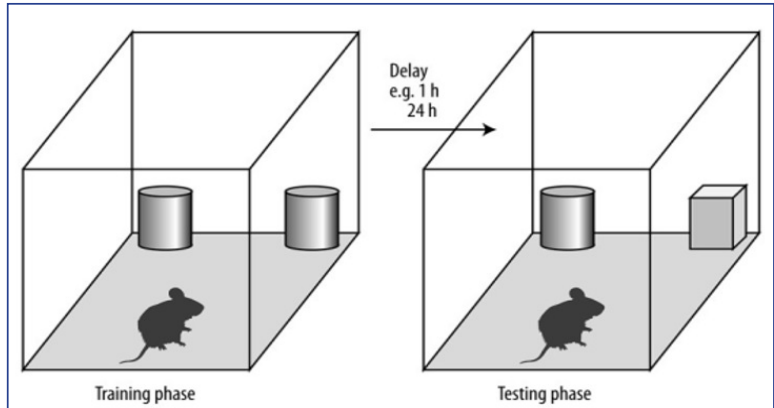

تصوير 1- آزمون تشخيص شئ جديد (•ه).

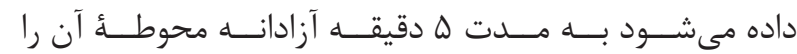

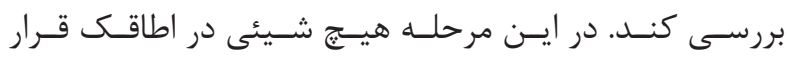

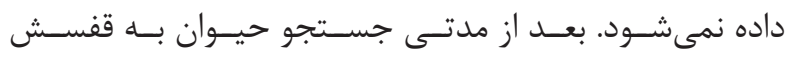

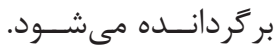

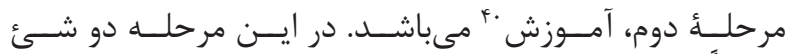

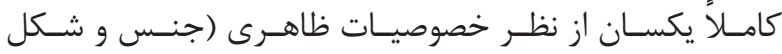

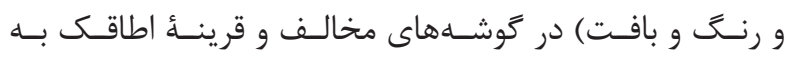

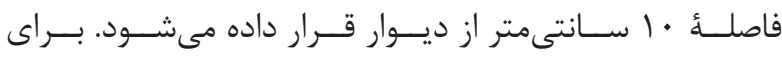

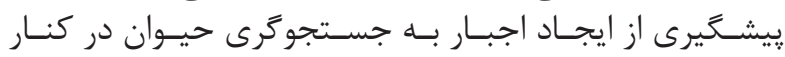

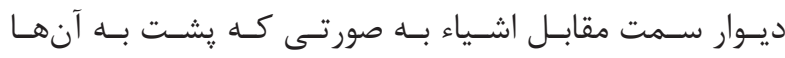

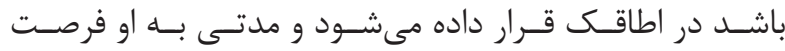

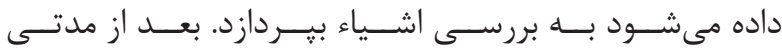

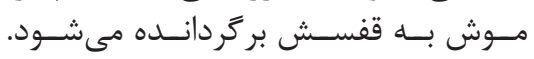

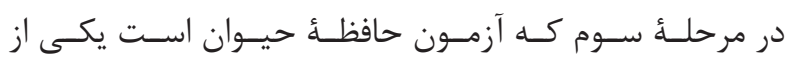

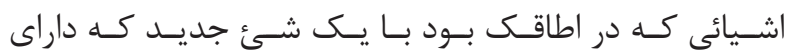

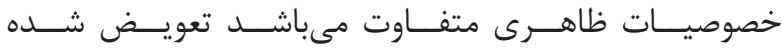

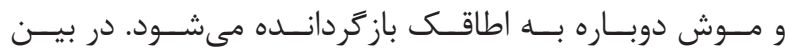

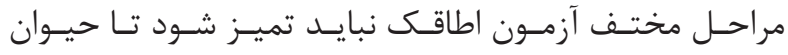

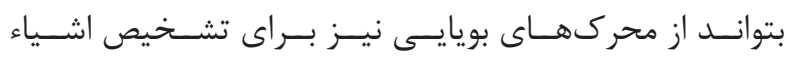

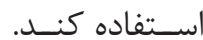

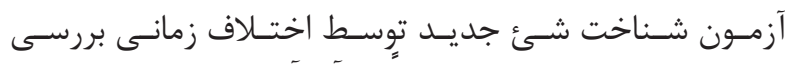

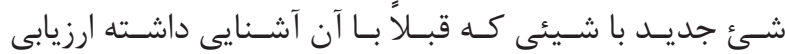

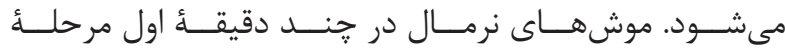

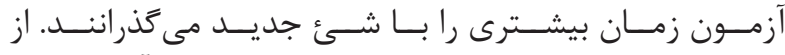

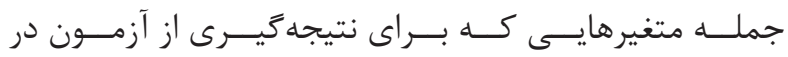

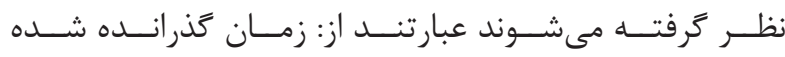

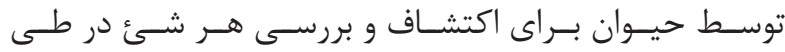

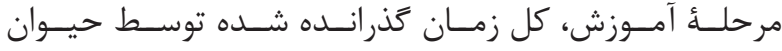

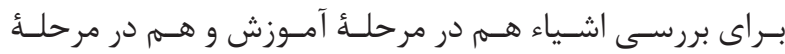

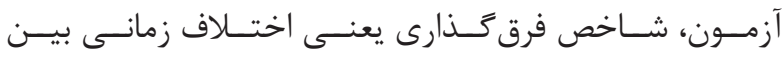

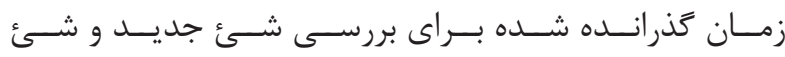

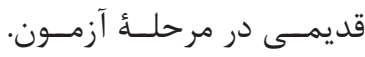

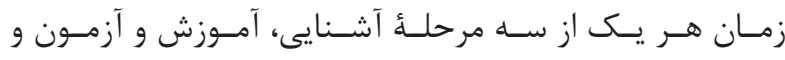

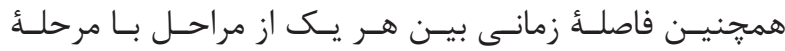

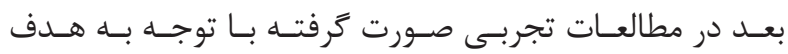

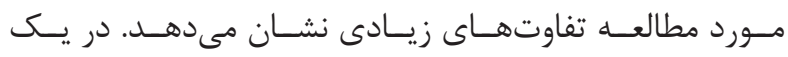

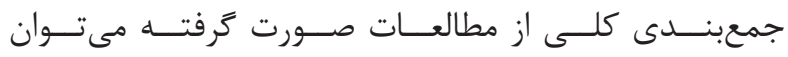




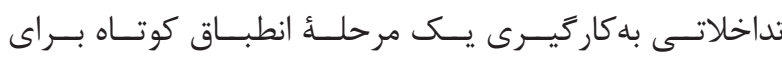

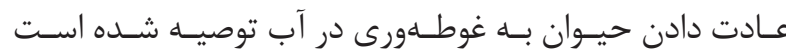

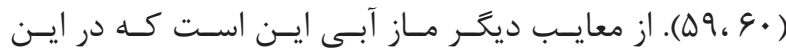

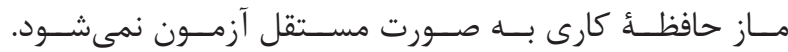

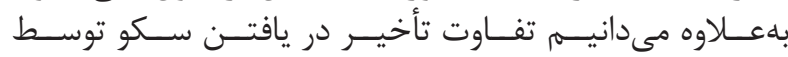

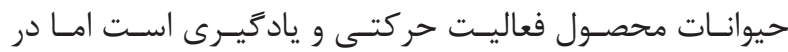

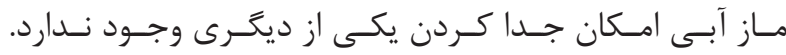

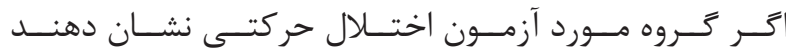

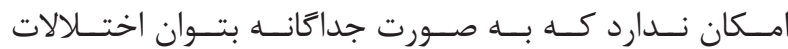

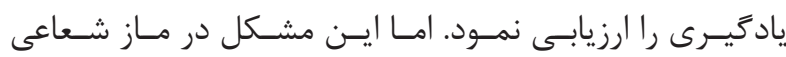

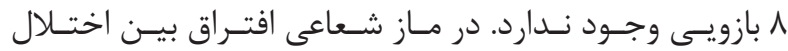

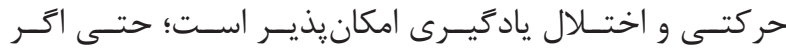

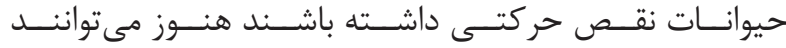

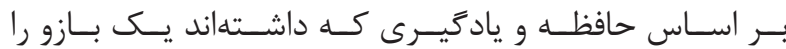

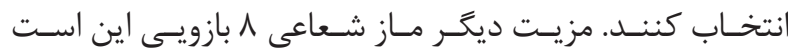

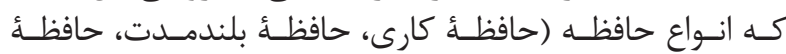

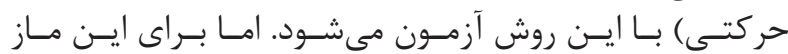

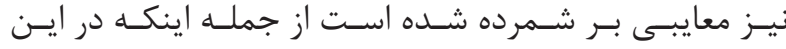

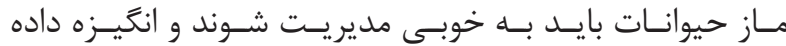

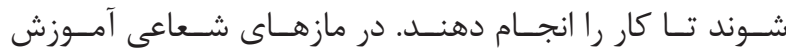

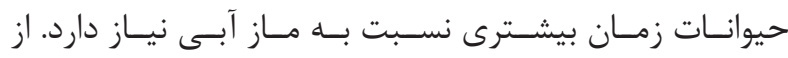

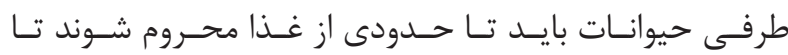

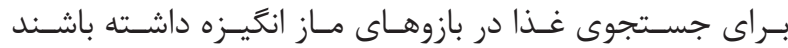

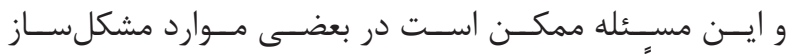

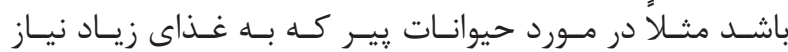

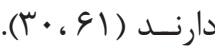

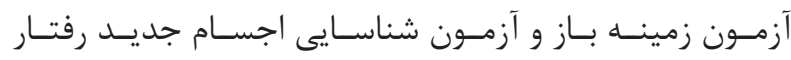

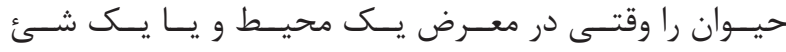

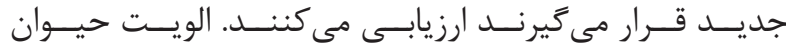

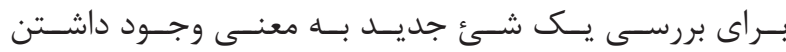

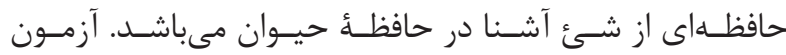

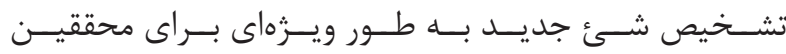

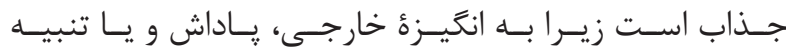

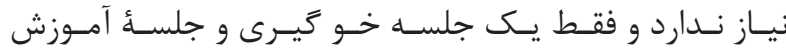

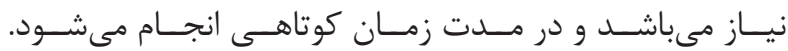

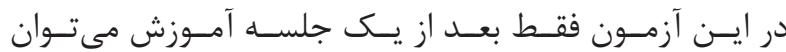

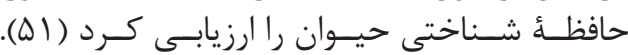

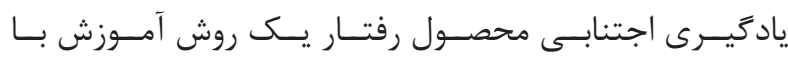

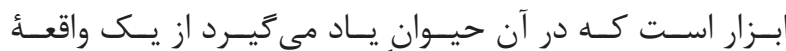

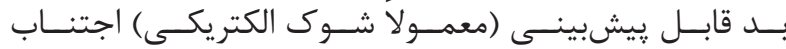

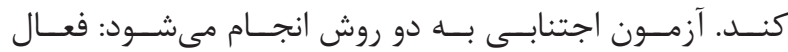

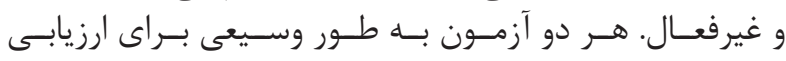

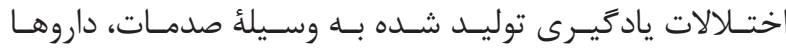

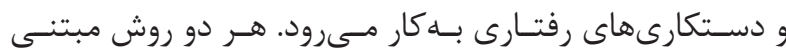

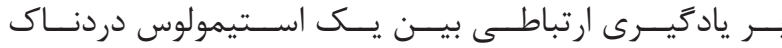

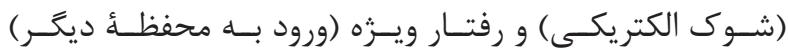

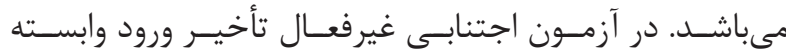

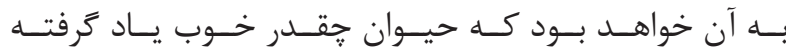

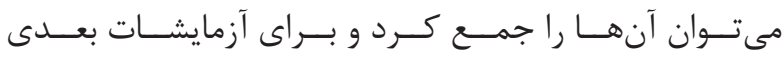

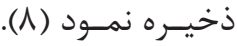

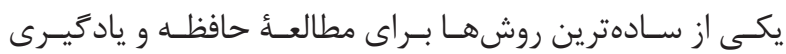

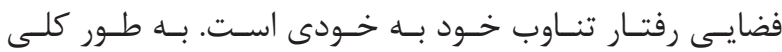

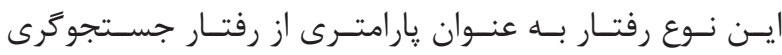

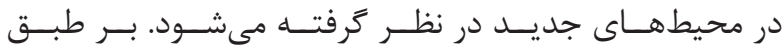

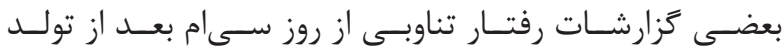

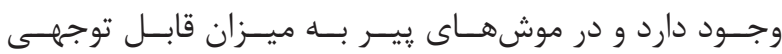

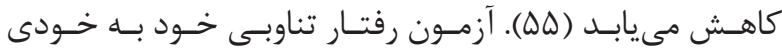

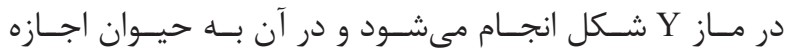

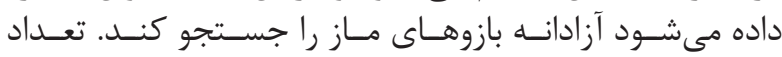

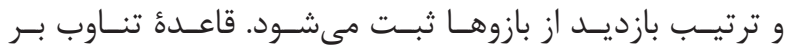

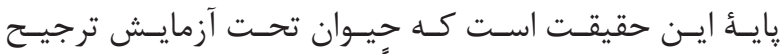

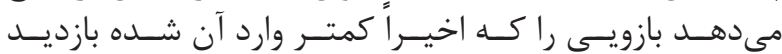

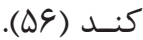

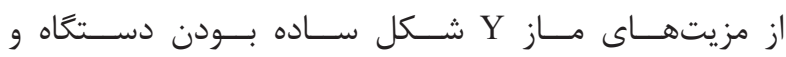

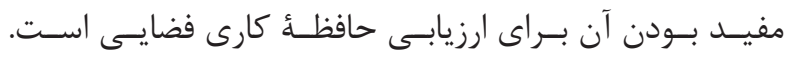

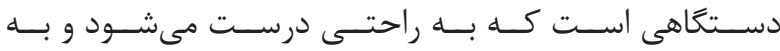

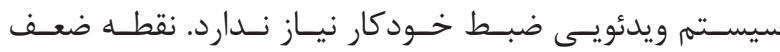

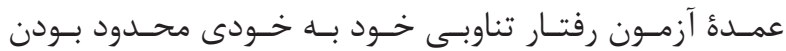

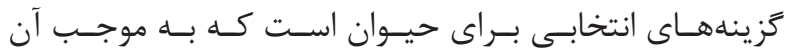

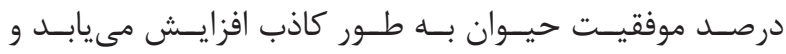

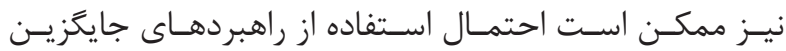

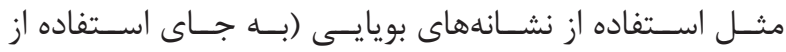

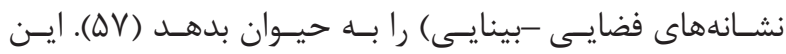

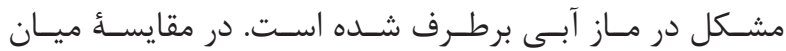

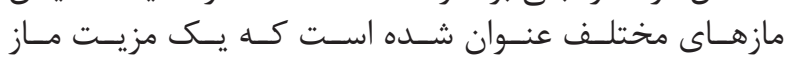

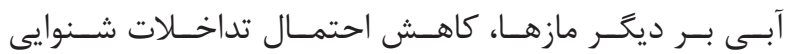

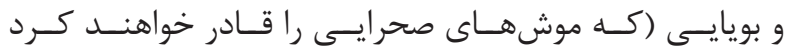

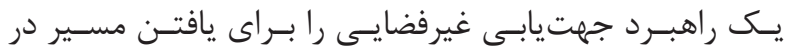

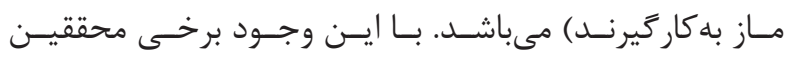

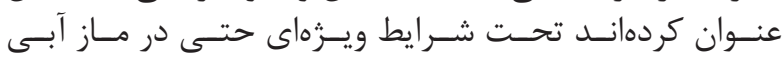

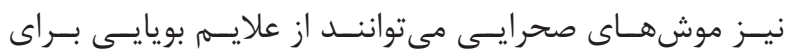

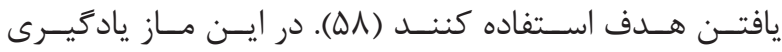

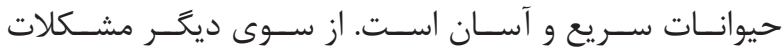

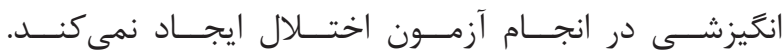

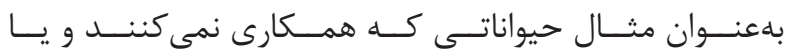

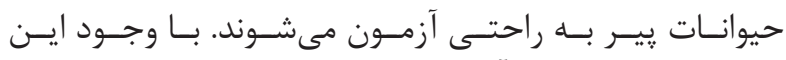

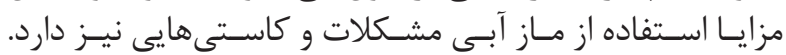

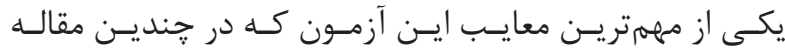

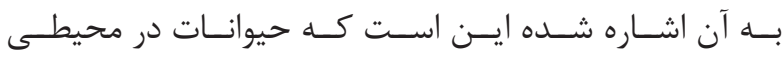

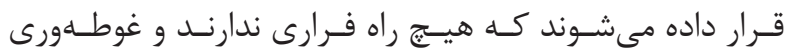

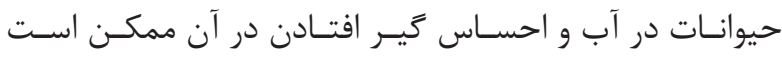

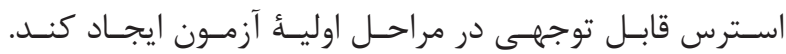

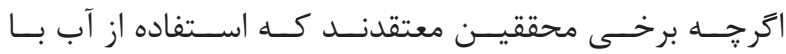

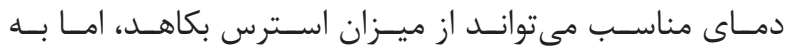

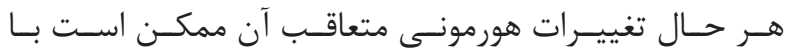

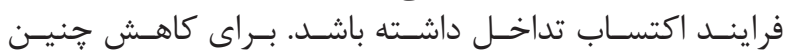




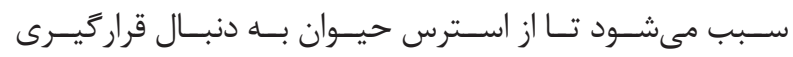

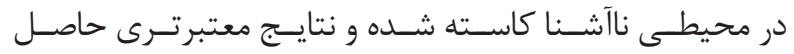

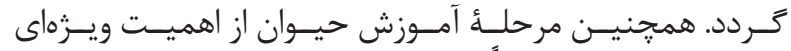

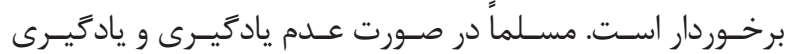

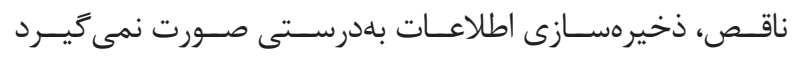

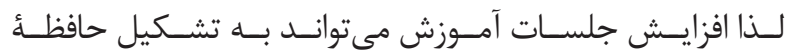

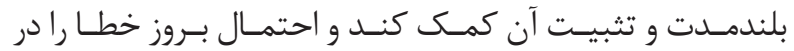

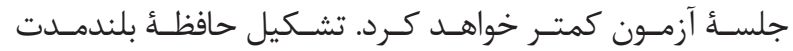

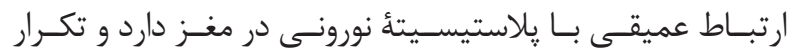

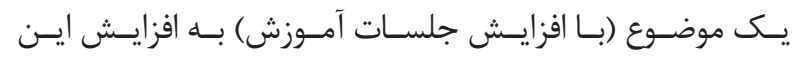

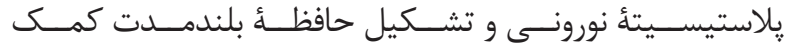

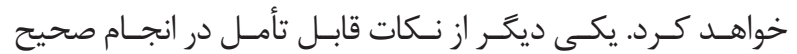

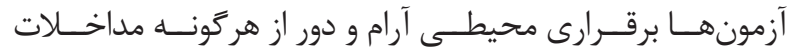

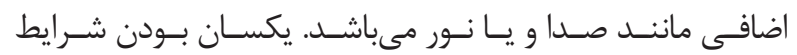

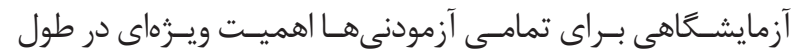

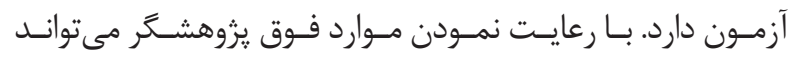

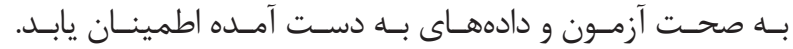

1. Levy MN, Berne RM, Koeppen BM, Stanton BA. Berne $\&$ levy principles of physiology. $4^{\text {th }}$ ed. Mosby. 2006.

2. Va S. Synopsis of psychiatry. Philadelphia: Lippincott Williams \& Wilkins. 2003.

3. Barrett KE, Barman SM, Boitano S, Brooks H. Ganong's review of medical physiology. NY: McGrawHill Medical. 2009.

4. The open field test the university of nairobi: www. uonbi.ac.ke/projects/ibro/.../open-field-method-2005. pdf. 2005.

5. Pietropaolo S. Mood and anxiety-related phenotypes in mice: characterization using behavioral tests.TD Gould. Genes, Brain and Behavior. 2010; 9(5): 544.

6. Ferreira FR, Spini VBMG, Lopes EJ, Lopes RFF, Moreira EA, Amaral MAF, et al. Effect of feed restriction on learning, memory and stress of rodents. Bioscience Journal. 2006; 22(1).

7. Vianna M, Izquierdo LA, Barros DM, De Souza M, Rodrigues C, Sant'Anna MK, et al. Pharmacological differences between memory consolidation of habituation to an open field and inhibitory avoidance learning. Braz J Med Biol Res. 2001; 34(2): 233-40.

8. Quillfeldt JA. Behavioral methods to study learning and memory in rats. Rodent Model as Tools in Ethical Biomedical Research. 2016; 271-311.

9. Dreier JP, Major S, Pannek HW, Woitzik J, Scheel M,
باشـد كـهـه وارد محفظـــ تاريـك نشــود. در مقابـل در آزمــون

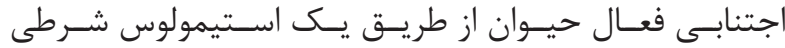

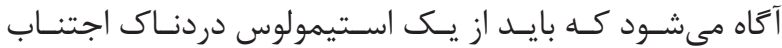

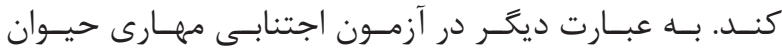

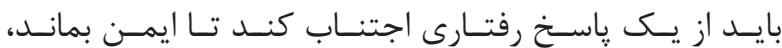

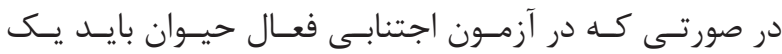

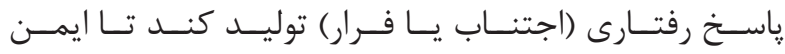

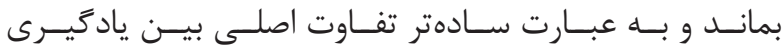

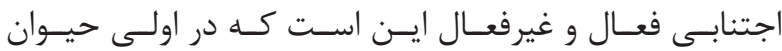

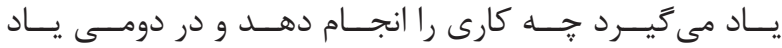

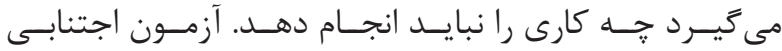

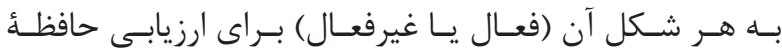

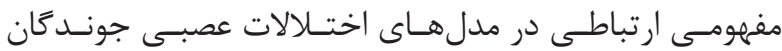

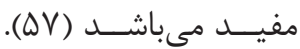

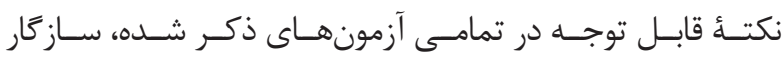

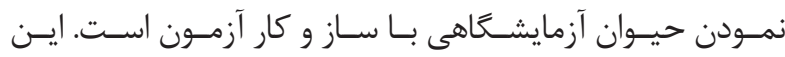

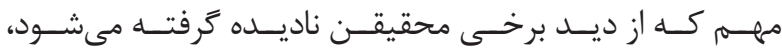

منابع

Wiesenthal D, et al. Spreading convulsions, spreading depolarization and epileptogenesis in human cerebral cortex. Brain. 2012; 135(1): 259-75

10. Sarnyai Z, Sibille EL, Pavlides C, Fenster RJ, McEwen BS, Tóth M. Impaired hippocampaldependent learning and functional abnormalities in the hippocampus in mice lacking serotonin 1A receptors. Proc Natl Acad Sci U S A. 2000; 97(26): 14731-6.

11. Baluchnejadmojarad T, Roghani M. Chronic epigallocatechin-3-gallate ameliorates learning and memory deficits in diabetic rats via modulation of nitric oxide and oxidative stress. Behav Brain Res. 2011; 224(2): 305-10.

12. Mirshekar M, Roghani M, Khalili M, Baluchnejadmojarad T. Chronic oral pelargonidin alleviates learning and memory disturbances in streptozotocin diabetic rats. Iran J Pharm Res. 2011; 10(3): $\quad 569-75$.

13. Jamali-Raeufy N, Roghani M, Ramazi S, Mansouri M. Administration of salvianolic acid B attenuates learning and memory deficits in diabetic rats: involvement of oxidative stress. Journal of Basic and Clinical Pathophysiology. 2014; 2(2): 43-50.

14. Roghani M, Khalili M, Baluchnejadmojarad T, Heydari A. The effect of hesperetin on short-term spatial memory and passive avoidance learning and memory in diabetic rats. Arak Medical University Journal (AMUJ). 2011; 14(1): 46-54.

15. Narwal S, Saini D, Kumari K, Narwal S, Singh G, 
Negi RS, et al. Behavior \& pharmacological animal models for the evaluation of learning \& memory condition. Indo Global J Pharm Sci. 2012; 2(2): 121-9.

16. Lotfinia M, Lotfinia AA, Asaadi S. Effect of sumatriptan on learning and memory impairment resulting from repetitive spreading depression. Shefaye Khatam. 2014; 2(3): 37-44.

17. Step-through passive avoidance. http://www.labthai. co.th/ugo-behaviour.html: LABTHAI SCIENTIFIC. 2017.

18. Zarrindast MR, Farajzadeh Z, Rostami P, Rezayof $A$, Nourjah P. Involvement of the ventral tegmental area (VTA) in morphine-induced memory retention in morphine-sensitized rats. Behavioural Brain Research. 2005; 163(1): 100-6.

19. Jamali-Raeufy N, Nasehi M, Zarrindast MR. Influence of N-methyl D-aspartate receptor mechanism on WIN55, 212-2-induced amnesia in rat dorsal hippocampus. Behav Pharmacol. 2011; 22(7): 645-54.

20. Klenerova V, Kaminsky O, Sida P, Hlinak Z, Krejci I, Hynie S. Impaired passive avoidance acquisition in Wistar rats after restraint/cold stress and/or stresscopin administration. Gen Physiol Biophys. 2003; 22(1): 115-20.

21. Nedaei SE, Pourmotabbed A, Aeenfar M, Seifi Z. The therapeutic effect of crocin on scopolamine induced retrograde amnesia in male rats. Journal of Kermanshah University of Medical Sciences (J Kermanshah Univ Med Sci). 2012; 16(2): 92-9.

22. Sousa DNd, Santana WMd, Ferreira VM, Duarte WR. Behavioural and cognitive effects of simvastatin dose used in stimulation of bone regeneration in rats. Acta Cirurgica Brasileira. 2014; 29(3): 151-7.

23. Izquierdo I, Furini CR, Myskiw JC. Fear memory. Physiological Reviews. 2016; 96(2): 695-750.

24. Vinadé ER, Schmidt AP, Frizzo ME, Portela LV, Soares FA, Schwalm FD, et al. Effects of chronic administered guanosine on behavioral parameters and brain glutamate uptake in rats. J Neurosci Res. 2005; 79(1-2): 248-53.

25. Borba Filho GL, Zenki KC, Kalinine E, Baggio S, Pettenuzzo L, Zimmer ER, et al. A new device for stepdown inhibitory avoidance task-effects of low and high frequency in a novel device for passive inhibitory avoidance task that avoids bioimpedance variations. PloS One. 2015; 10(2): e0116000.

26. Rubio S, Miranda R, Cuesta M, Begega A, Santín LJ,
Arias JL. Active avoidance conditioning in rats: absence of sex difference and estrous effect. Psicothema. 1999; 11(3): 655-61.

27. Behavioral assessments in rodents. http://www. radiantthinking.us/memory-theory/ii-behavioralassessments-in-rodents.html2015 [updated 03 Dec 2015 ].

28. Dimitrova D, Getova D. Comparison the effects of tacrine and galantamine on active avoidance test in rats with diazepam-amnesia model. Science and Technologies. 2015; 5(1): 405-10.

29. McNamara RK, Skelton RW. The neuropharmacological and neurochemical basis of place learning in the Morris water maze. Brain Res Brain Res Rev. 1993; 18(1): 33-49.

30. D’Hooge R, De Deyn PP. Applications of the Morris water maze in the study of learning and memory. Brain Res Brain Res Rev. 2001; 36(1): 60-90.

31. Ebrahimi S, Rashidy-Pour A, Vafaei AA, Akhavan MM. Central $\beta$-adrenergic receptors play an important role in the enhancing effect of voluntary exercise on learning and memory in rat. Behav Brain Res. 2010; 208(1): 189-93.

32. Morris water maze (MWM). http://www.augusta. edu/core/labs/sabc/morriswatermaze.php: Augusta University. 2016.

33. Saffarzadeh F, Eslamizade MJ, Hosseini M. The effect of L-Arginine on spatial memory in ovariectomized rats. Shefaye Khatam. 2013; 1(1): 13-8.

34. Golestani S, Edalatmanesh MA, Hosseini M. The effects of sodium valproate on learning and memory processes in trimethyltinmodel of alzheimer's disease. Shefaye Khatam. 2014; 2(3): 19-26

35. Vorhees CV, Williams MT. Morris water maze: procedures for assessing spatial and related forms of learning and memory. Nature Protocols. 2006; 1(2): 848-58.

36. Morris R. Developments of a water-maze procedure for studying spatial learning in the rat. Journal of Neuroscience Methods. 1984; 11(1): 47-60

37. Morris RG. Morris water maze. Scholarpedia. 2008; 3(8): 6315 .

38. Olton DS, Collison C, Werz MA. Spatial memory and radial arm maze performance of rats. Learning and Motivation. 1977; 8(3): 289-314

39. Radial Arm Maze. https://www.uwrf.edu/PSYC/ 


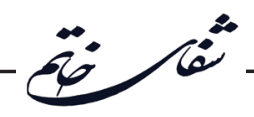

StudentInformation/Conditioning_Lab.cfm: University of Wisconsin-River Falls; 2016.

40. Dubreuil D, Tixier C, Dutrieux G, Edeline J-M. Does the radial arm maze necessarily test spatial memory? Neurobiology of Learning and Memory. 2003; 79(1): 109-17.

41. Tarragon E, Lopez L, Ros-Bernal F, Yuste J, OrtizCullera V, Martin E, et al. The Radial arm maze (ram) for the evaluation of working and reference memory deficits in the diurnal rodent octodon degus. Proc Meas Behav. 2012; 98-100.

42. Stuchlik A, Petrasek T, Hatalová H, Rambousek L, Nekovarova T, Vales K. Behavioral tests for evaluation of information processing and cognitive deficits in rodent animal models of neuropsychiatric disorders. Schizophrenia in the 21st century. 2012: 153-80.

43. Schaar KL, Brenneman MM, Savitz SI. Functional assessments in the rodent stroke model. Experimental \& Translational Stroke Medicine. 2010; 2(1): 13. doi. org/10.1186/2040-7378-2-13.

44. Hodges H. Maze procedures: the radial-arm and water maze compared. Cognitive Brain Research. 1996; 3(3): 167-81.

45. Stevens R. Scopolamine impairs spatial maze performance in rats. Physiology \& Behavior. 1981; 27(2): 385-6.

46. Ennaceur A, Delacour J. A new one-trial test for neurobiological studies of memory in rats. 1: behavioral data. Behavioural Brain Research. 1988; 31(1): 47-59.

47. Goulart B, De Lima M, De Farias C, Reolon G, Almeida V, Quevedo J, et al. Ketamine impairs recognition memory consolidation and prevents learning-induced increase in hippocampal brain-derived neurotrophic factor levels. Neuroscience. 2010; 167(4): 969-73.

48. Silvers JM, Harrod SB, Mactutus CF, Booze RM. Automation of the novel object recognition task for use in adolescent rats. Journal of Neuroscience Methods. 2007; 166(1): 99-103.

49. Piterkin P, Cole E, Cossette M-P, Gaskin S, Mumby DG. A limited role for the hippocampus in the modulation of novel-object preference by contextual cues. Learning \& Memory. 2008; 15(10): 785-91.
50. Brodziak A, Kołat E, Różyk-Myrta A. In search of memory tests equivalent for experiments on animals and humans. Med Sci Monit. 2014; 20: 2733-9.

51. Antunes M, Biala G. The novel object recognition memory: neurobiology, test procedure, and its modifications. Cognitive Processing. 2012; 13(2): 93110 .

52. Bevins RA, Besheer J. Object recognition in rats and mice: a one-trial non-matching-to-sample learning task to study'recognition memory'. Nature Protocols. 2006; 1(3): 1306-11.

53. Okano H, Hirano T, Balaban E. Learning and memory. Proceedings of the National Academy of Sciences. 2000; 97(23): 12403-4.

54. Baxter MG. "I've seen it all before": explaining agerelated impairments in object recognition. Theoretical Comment on Burke et al. 2010. doi.org/10.1037/ a0021029.

55. Lalonde R. The neurobiological basis of spontaneous alternation. Neuroscience \& Biobehavioral Reviews. 2002; 26(1): 91-104.

56. Thinus-Blanc C, Save E, Poucet B. Animal spatial cognition and exploration. A handbook of spatial research paradigms and methodologies. 1998; 2: 59-86.

57. Paul CM, Magda G, Abel S. Spatial memory: theoretical basis and comparative review on experimental methods in rodents. Behav Brain Res. 2009; 203(2): 151-64.

58. Means LW, Alexander SR, O’Neal MF. Those cheating rats: male and female rats use odor trails in a water-escape "working memory" task. Behav Neural Biol. 1992; 58(2): 144-51.

59. Block F. Global ischemia and behavioural deficits. Prog Neurobiol. 1999; 58(3): 279-95.

60. Wenk GL. Assessment of spatial memory using the radial arm maze and Morris water maze. Current Protocols in Neuroscience. 2004; 5-8.

61. Hölscher C, O’Mara S. Model learning and memory systems in neurobiological research: conditioning and associative learning procedures and spatial learning paradigms. Neuroscience Labfax: Academic Press London: 1997; p. 81-93. 